\title{
Hospital admissions and place of death of residents of care homes receiving specialist healthcare services: A systematic review without meta-analysis
}

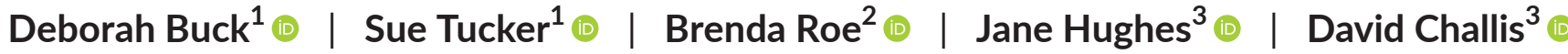

${ }^{1}$ Social Care and Society, University of Manchester, Manchester, UK

${ }^{2}$ Faculty of Health, Social Care and Medicine, Edge Hill University, Ormskirk, UK

${ }^{3}$ Institute of Mental Health, University of Nottingham, Nottingham, UK

\section{Correspondence}

Deborah Buck, Social Care and Society, University of Manchester, Oxford Road, Manchester, M13 9PL, UK.

Email: debbiebuck001@gmail.com

\section{Funding information}

This article presents independent research funded by the National Institute for Health Research (NIHR) School for Social Care Research (SSCR) (Funding reference number C088/CM/UMDC-P113: Effective Healthcare Support to Care Homes). The views expressed in this article are those of the authors and not necessarily those of the NIHR SSCR or the Department of Health and Social Care, NIHR or NHS.

\begin{abstract}
Aim: To synthesize evidence on the ability of specialist care home support services to prevent hospital admission of older care home residents, including at end of life.

Design: Systematic review, without meta-analysis, with vote counting based on direction of effect.

Data sources: Fourteen electronic databases were searched from January 2010 to January 2019. Reference lists of identified reviews, study protocols and included documents were scrutinized for further studies.

Review methods: Papers on the provision of specialist care home support that addressed older, long-term care home residents' physical health needs and provided comparative data on hospital admissions were included. Two reviewers undertook study selection and quality appraisal independently. Vote counting by direction of effect and binomial tests determined service effectiveness.

Results: Electronic searches identified 79 relevant references. Combined with 19 citations from an earlier review, this gave 98 individual references relating to 92 studies. Most were from the UK (22), USA (22) and Australia (19). Twenty studies were randomized controlled trials and six clinical controlled trials. The review suggested interventions addressing residents' general health needs $(p<.001)$, assessment and management services $(p<.0001)$ and non-training initiatives involving medical staff $(p<.0001)$ can reduce hospital admissions, while there was also promising evidence for services targeting residents at imminent risk of hospital entry or post-hospital discharge and training-only initiatives. End-of-life care services may enable residents to remain in the home at end of life $(p<.001)$, but the high number of weak-rated studies undermined confidence in this result.

Conclusion: This review suggests specialist care home support services can reduce hospital admissions. More robust studies of services for residents at end of life are urgently needed. Impact: The review addressed the policy imperative to reduce the avoidable hospital admission of older care home residents and provides important evidence to inform service design. The findings are of relevance to commissioners, providers and residents.
\end{abstract}


KEYWORDS

care homes, hospital admissions, long-term care facilities, nursing homes, older people, outcomes, place of death, specialist health services, systematic review without meta-analysis,

vote counting

\section{1 | INTRODUCTION}

The number of care homes in the UK (variously known as nursing homes or long-term care/residential/aged care/assisted living facilities in different countries) has declined in recent years. Nevertheless, over 400,000 older adults live in such facilities and despite the ongoing imperative to provide care at home, population ageing is likely to increase demand for care home beds in coming decades, a situation seen internationally (Deloitte Access Economics, 2020; HarrisKojetin et al., 2019; Nuffield Trust, 2020).

Care home residents are typically frail with multiple physical and mental co-morbidities and subject to polypharmacy (BGS, 2016; Gordon et al., 2014; Russell, 2017). However, care homes often find it difficult to access timely and appropriate healthcare support for residents, resulting in the under-detection of potentially treatable conditions, increased hospital admissions and lack of resident choice about place of death (Iliffe et al., 2016; NSW Aged Care Roundtable, 2019; NHS England, 2015). Although hospital admission of older adults is sometimes appropriate, it is often associated with adverse effects including infection, delirium, confusion and falls (Agotnes et al., 2016; Arendts et al., 2017; McAndrew et al., 2016). It is thus concerning that up to two-fifths of emergency admissions of older care home residents could be managed in the care home or prevented with better care or vigilance (NHS, 2019; Wolters et al., 2019). Furthermore, although most care home residents would prefer to remain in the care home at end of life (Ennis et al., 2015; Finucane et al., 2013; Wiggins et al., 2019), around 30\% die in hospital (Public Health England, 2017).

There is increasing policy attention given to this issue. The NHS Long-Term Plan acknowledged the need to improve care home support (NHS, 2019), while the 'Framework for Enhanced Health in Care Homes' highlighted the need for equivalent services to people at home. One service specification for primary care networks (groups of local general practices) relates to enhanced care for care homes and others are relevant, including structured medication reviews and anticipatory care (NHS England, 2020).

\section{1 | Background}

Historically, a patchwork of specialist healthcare support services for care homes has emerged. For the purpose of this review, these are defined as services whereby healthcare professionals, with time dedicated to this role, provide specialist clinical support to identify and address residents' healthcare needs (Tucker et al., 2019). Some enhance standard primary care, allocating named practitioners to specific care homes or supplementing medical care with specialist nurse input; others involve partnerships between primary and secondary care and some are led by pharmacists. Core activities include comprehensive assessment of new residents; regular, structured multidimensional reviews; falls prevention; medication reviews; advance care planning and end-oflife care.

Evidence of a relationship between the provision of specialist healthcare support services and reduced avoidable hospital admissions would provide a strong argument for their expansion. However, the extent to which current models achieve this is unclear. A previous review (Clarkson et al., 2018) explored the organization, activities and responsibilities of specialist healthcare support services, but did not systematically explore outcomes. Other reviews have explored the extent to which specific activities including optimizing prescribing (Alldred et al., 2016; Forsetlund et al., 2011; Thiruchelvam et al., 2017; Wallerstedt et al., 2014), antibiotic stewardship programmes (Feldstein et al., 2018) and palliative care initiatives (Hall et al., 2011) can reduce admissions. However, the quality of evidence in these and a review of interventions to reduce acute admissions of nursing home residents (Graverholt et al., 2014) were generally poor, while the heterogeneity of interventions, methods and outcomes in reviews of the effectiveness of input from staff with geriatric expertise, integrated and partnership working (Davies et al., 2011; Goldman, 2013; Santosaputri et al., 2018) precluded robust conclusions. As such, an overall picture of the evidence to inform service development is lacking.

In addressing this gap, this review employed two taxonomies (Table 1) developed from a UK survey of specialist healthcare support services (Hargreaves et al., 2019). The first has five categories, using constructs of specialist support identified in the care home literature (Clarkson et al., 2018). The second allocated services with similar characteristics into three subgroups using latent class analysis (Hargreaves et al., 2019).

\section{THE REVIEW}

\section{1 | Aims}

The primary aim was to identify, appraise and synthesize the evidence on the ability of specialist healthcare support services to: (1) reduce hospital admissions of older care home residents and (2) enable them to remain in the care home at end of life. Secondary aims were to: (3) determine whether particular service subgroups produced different outcomes; (4) explore how usual/standard care was defined and (5) identify service costs. 
TABLE 1 Taxonomies of specialist healthcare support

\begin{tabular}{|c|c|c|c|}
\hline \multicolumn{2}{|l|}{ Taxonomy 1 (Clarkson et al., 2018) } & \multicolumn{2}{|c|}{ Taxonomy 2 (Hargreaves et al., 2019) } \\
\hline Category & Brief description & Category & Brief description \\
\hline $\begin{array}{l}\text { Assessment, no medical } \\
\text { practitioner involvement }\end{array}$ & $\begin{array}{l}\text { Provide assessment only. Advice and/or } \\
\text { direct training may be given to care } \\
\text { home staff and/or recommendations } \\
\text { made to residents' primary care } \\
\text { physicians. No on-going management } \\
\text { or support of individual care home } \\
\text { residents or additional input from } \\
\text { physicians (over usual care). }\end{array}$ & $\begin{array}{l}\text { Predominantly direct } \\
\text { care }\end{array}$ & $\begin{array}{l}\text { High probability of scheduled input } \\
\text { and support for all residents. } \\
\text { Moderate probability of } \\
\text { medication management. Low } \\
\text { probability of staff training. }\end{array}$ \\
\hline $\begin{array}{c}\text { Assessment, (additional) medical } \\
\text { practitioner involvement }\end{array}$ & $\begin{array}{l}\text { Assessment only as above, but additional } \\
\text { input from physicians (over usual } \\
\text { care). }\end{array}$ & $\begin{array}{l}\text { Predominantly } \\
\text { indirect care }\end{array}$ & $\begin{array}{l}\text { Low probability of scheduled input } \\
\text { and support for all residents and } \\
\text { medication management. High } \\
\text { probability of staff training. }\end{array}$ \\
\hline $\begin{array}{l}\text { Assessment and management, } \\
\text { no medical practitioner } \\
\text { involvement }\end{array}$ & $\begin{array}{l}\text { Provides assessment and on-going } \\
\text { management of the health/care } \\
\text { of residents (potentially including } \\
\text { prescribing). No additional input from } \\
\text { physicians (over usual care). Advice } \\
\text { and/or direct training may be given to } \\
\text { care home staff. }\end{array}$ & $\begin{array}{l}\text { Direct and indirect } \\
\text { care }\end{array}$ & $\begin{array}{l}\text { Moderate-to-high probability of } \\
\text { scheduled input, support for all } \\
\text { residents, medication management } \\
\text { and staff training. }\end{array}$ \\
\hline $\begin{array}{l}\text { Assessment and management, } \\
\text { medical practitioner } \\
\text { involvement }\end{array}$ & $\begin{array}{l}\text { Assessment and management as above, } \\
\text { including additional input from } \\
\text { physicians (over usual care). }\end{array}$ & & \\
\hline Training and support & $\begin{array}{l}\text { Support provided to the home, not } \\
\text { individual residents. This may include } \\
\text { training for care home staff and/or } \\
\text { assistance with quality improvement } \\
\text { initiatives. However, the team do not } \\
\text { assess or see individual residents. }\end{array}$ & & \\
\hline
\end{tabular}

\section{2 | Design}

A systematic review, without meta-analysis, with vote counting based on direction of effect updated and extended the review by Clarkson et al. (2018) in seeking studies published post 2010 and appraising outcomes. The review followed established guidance (CRD, 2009; Rutter et al., 2010) and the protocol was registered (PROSPERO) and published (Tucker et al., 2019). Reporting used the SWiM reporting guideline (Campbell et al., 2020) in conjunction with the 2020 PRISMA checklist (Page et al., 2021) to promote transparency.

\section{3 | Search methods}

Searches were conducted in 14 electronic databases: Agelnfo, CINAHL Plus, the Cochrane Database of Systematic Reviews, the Database of Abstracts of Reviews and Effectiveness, EMBASE (incorporating Medline), the Health Technology Assessment Database, Health Management Information Consortium, the Joanna Briggs Foundation, the National Health Service Economic Evaluation Database, PsycINFO, PubMed, PROSPERO, Social Care Online and Web of Science. Initial searches were undertaken in November 2017 and auto-alerts monitored until January 2019. Reference lists of identified reviews, included documents and study protocols, were scrutinized for further studies.

Four search blocks (endorsed by a research librarian) were combined in EMBASE and adjusted for other databases. These pertained to: (1) care homes; (2) healthcare; (3) older people and (4) hospital admission and place of death. An example search strategy for EMBASE is given in Supplementary Appendix S1. No geographical limitations were imposed, but non-English language publications were excluded.

\subsection{Study selection and quality appraisal}

Two reviewers (DB and $\mathrm{ST}$ ) independently screened the results against the study inclusion/exclusion criteria (Box 1) and quality appraised inclusions using the Effective Public Health Practice Project (EPHPP) Quality Assessment Tool (Armijo-Olivo et al., 2012; Thomas et al., 2004). This is suitable for quantitative studies and covers six domains (selection bias, study design, confounders, blinding, data collection and withdrawals/dropouts), each of which is rated strong, moderate or weak. A global rating is allocated depending on the number of weak ratings - strong (none), moderate (one) or weak (two plus) (Armijo-Olivo et al., 2012; Jackson \& Waters, 2005; Thomas et al., 2004). Given the 


\section{BOX 1 Review Inclusion and Exclusion Criteria}

\section{Studies}

Included: Empirical research studies and service descriptions published in peer-reviewed journals, in English language, that provided comparative quantitative data on the primary outcomes.

Excluded: Commentaries, opinion pieces and descriptive articles lacking empirical data; and studies not published in English language.

\section{Settings}

Included: Care homes for older people with or without nursing, including facilities for older people with dementia.

Excluded: Studies concerned solely with the delivery of care in hospital, individuals' own homes or other community settings.

\section{Participants}

Included: Older people, with or without dementia, permanently resident in care homes. Specifically, people aged 60 or over, samples with a mean/median age of 69 plus where age is reported, and samples described as samples of older people where age is not reported.

Excluded: Studies concerned only with the provision of support for younger or short-stay residents.

\section{Interventions}

Included: Studies of specialist services specifically designed to address the physical healthcare needs of older long-stay care home residents, including organizational or multi-faceted interventions with this goal.

Excluded: Services/interventions designed to address care home residents' mental health or social care needs, including those focused on the provision of person-centred care.

\section{Outcomes}

Included: Studies that reported information on ED transfers, hospital admissions (all admissions, emergency admissions, planned admissions and/or readmissions) and/or place of resident death.

Excluded: Studies that lacked relevant outcome data or which retrospectively explored associations in large administrative datasets (rather than evaluating particular services/interventions).

largely objective nature of the primary outcomes (raising fewer problems with reliability/validity than, say, clinical assessments), sections concerning the blinding of outcome assessors and data collection were adapted. (The modified tool is available from the authors.)

\subsection{Data extraction}

One reviewer extracted data on study ID, aims, design, methods, participants, interventions/services, comparison interventions/services, outcomes and costs using a specially designed Excel form. A second reviewer checked all extracted data for accuracy, consistency and agreement. Disagreements or uncertainties were resolved through discussion with the wider research group. The form was tested and refined by the same two reviewers and agreed by the review team before full data extraction. Where multiple reports were identified from the same study, information was extracted from each publication separately but combined into one study record for the purpose of synthesis.

\section{6 | Data synthesis}

The key characteristics and results of each study were charted, described and synthesized according to the primary outcomes. No studies were discarded because of their quality rating, but weakrated studies are highlighted in the text with the superscript*. Only studies that explicitly aimed to reduce hospitalization were included in effectiveness analyses.

A random-effects model meta-analysis (Borenstein et al., 2010) was attempted to estimate the effect size of specialist healthcare support interventions on hospitalizations. The analysis was conducted in Review Manager 5.4 (2020) and included 11 studies that reported the intervention and control group sample sizes and mean and standard deviation of hospital admissions/ED transfers post-intervention. Forest plots were presented to visualize the results. Sensitivity analyses explored the effects of study quality (excluding weak-rated studies) and outcome domains (excluding data on ED transfers).

Given the diversity of interventions, variation in effect measures and high level of missing/incomplete outcome estimates, 
FIGURE 1 PRISMA flow diagram

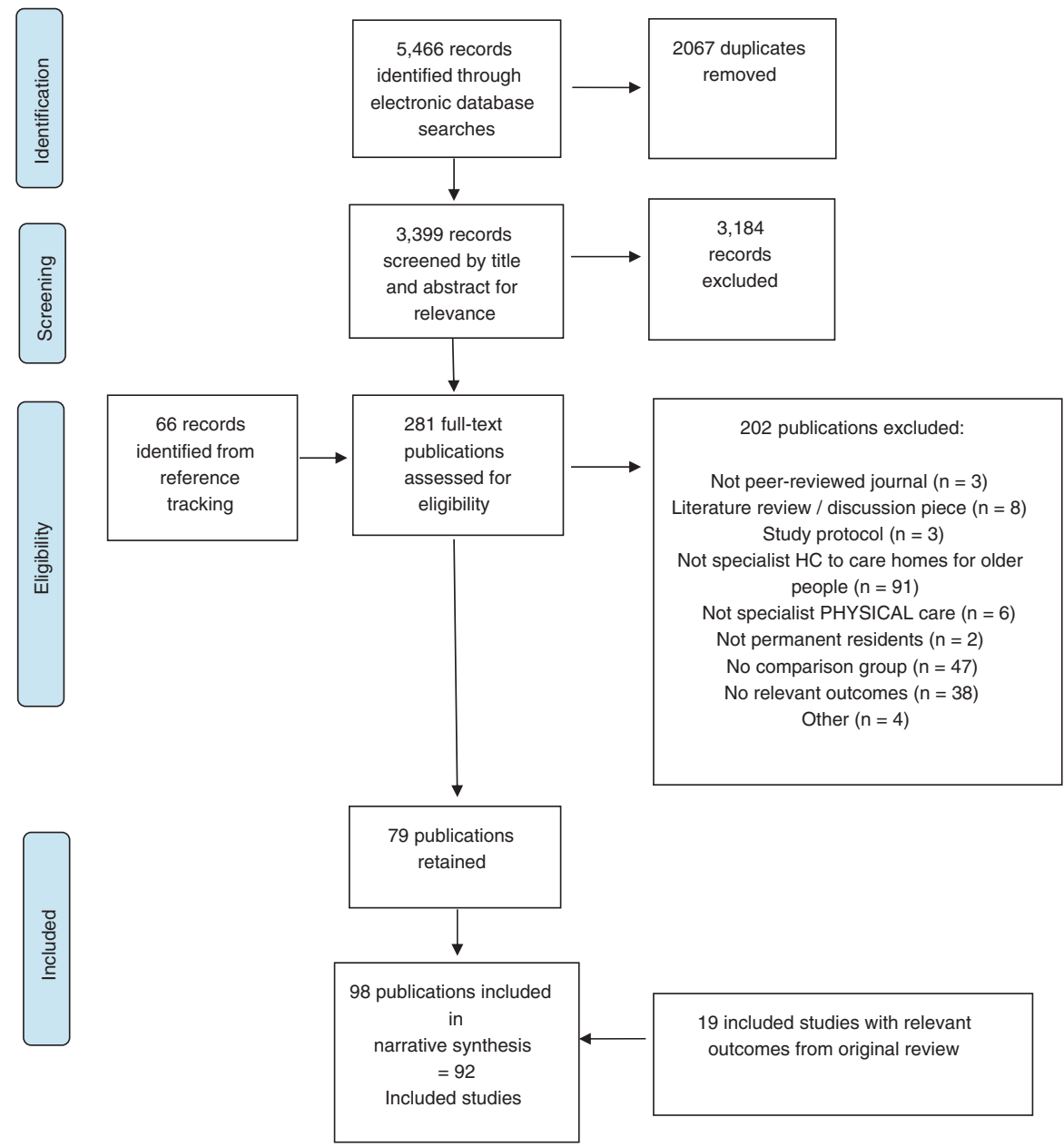

vote counting based on direction of effect (regardless of statistical significance or testing) was used to detect evidence of an effect for study subgroups according to the foci of intervention and two taxonomies (McKenzie \& Brennan, 2019). All studies reporting a positive effect on the number and/or rate of hospital admissions/ED transfers were considered beneficial regardless of any co-existing negative effects so as not to penalize studies reporting multiple outcomes. Studies reporting no change were classed as 'no positive outcome/not beneficial'; studies reporting practitioners' perceptions of avoided admissions or only hospital bed days were excluded. Binomial tests assessed statistical significance of the vote counting findings (McKenzie \& Brennan, 2019) and sensitivity analysis explored the effect of treating studies with mixed findings as negative (reversing their handling in the main analysis). Correction for multiple testing was undertaken using the conservative Bonferroni method of multiplying the $p$-values by the number of comparisons (Jafari \& Ansari-Pour, 2019) in cases where there appeared to be a significant outcome. This was applied to each subset of analyses, that is, considering foci of intervention as one set (six comparisons), and the models of specialist support, namely Taxonomy 1 and Taxonomy 2, as two further sets (five and three comparisons respectively).

\section{3 | RESULTS}

\subsection{Search outcomes}

Electronic database searches identified 5466 references (Figure 1). After duplicates were removed, 3399 records were screened by title and abstract for relevance and the full texts of the 215 remaining documents, plus 66 citations identified by reference tracking, were independently assessed against the inclusion/exclusion criteria. This resulted in 92 included studies (98 references): 79 from the searches plus 19 from the review by Clarkson et al. (2018).

\section{2 | Study characteristics}

Of the 92 included studies, 22 were undertaken in the UK, 22 in the USA and 19 in Australia (Tables 2 and 3). The earliest was published in 1991, but most $(n=82)$ were published between 2010 and 2019 . Supplementary Appendix S2 provides more detail.

Twenty studies were randomized controlled trials, six controlled clinical trials, 16 cohort analytic studies (two- or three-group pre and post) and 30 cohort studies (one group pre and post). Other designs included eleven (two or three groups) observational cohorts and six descriptive 


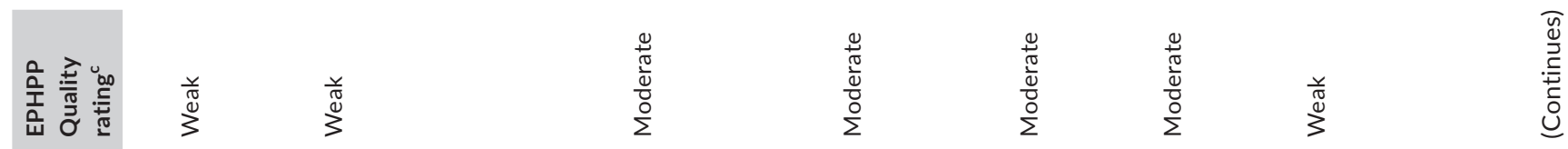

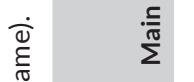

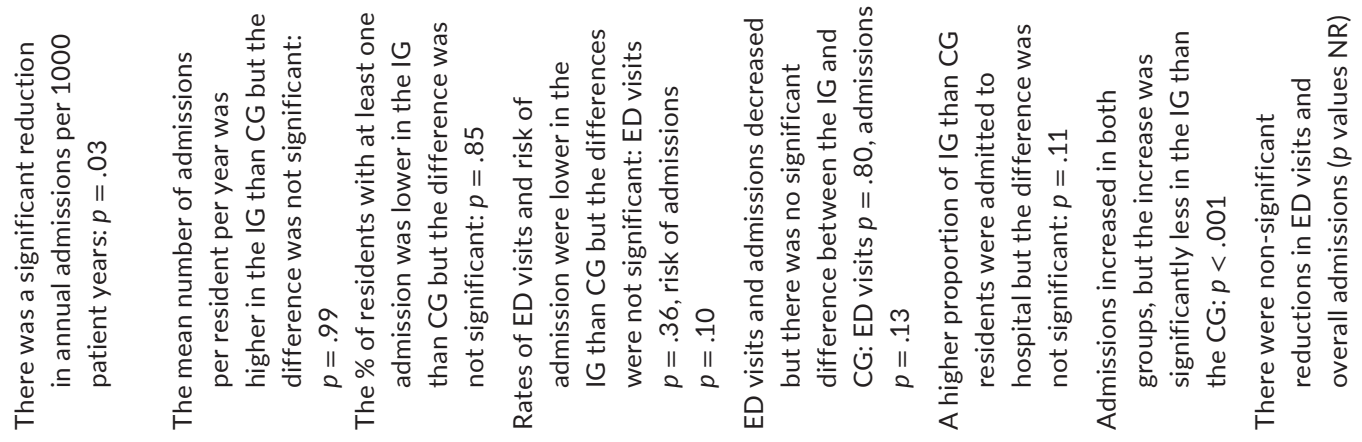
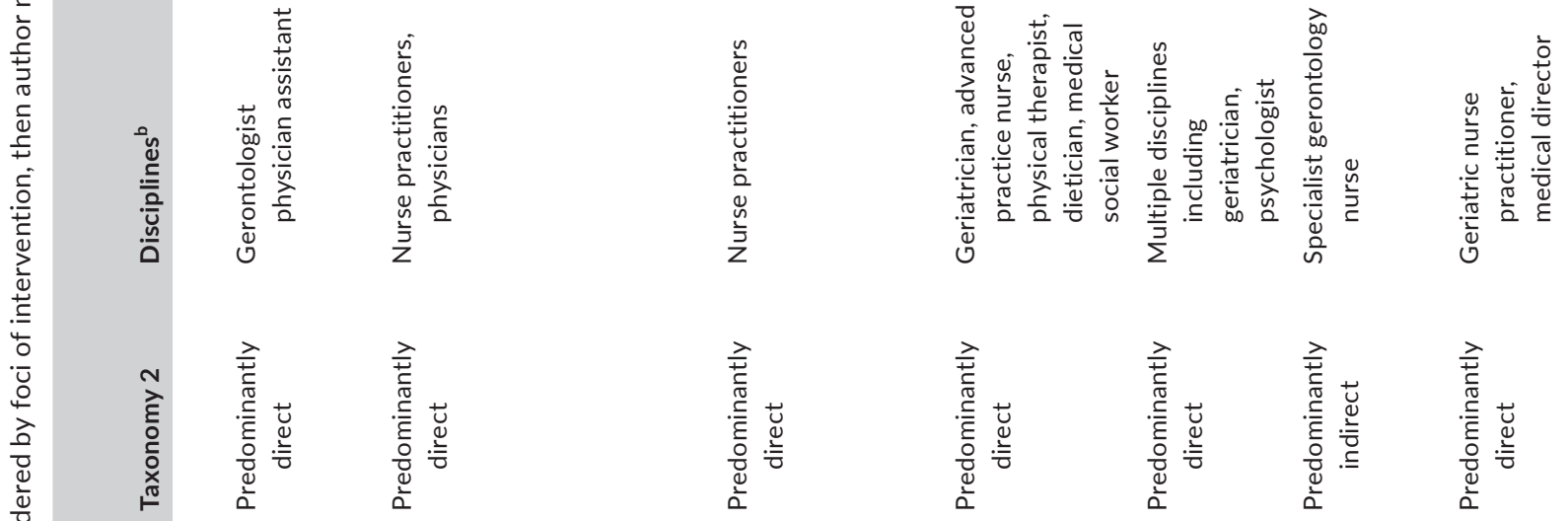

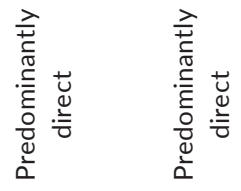
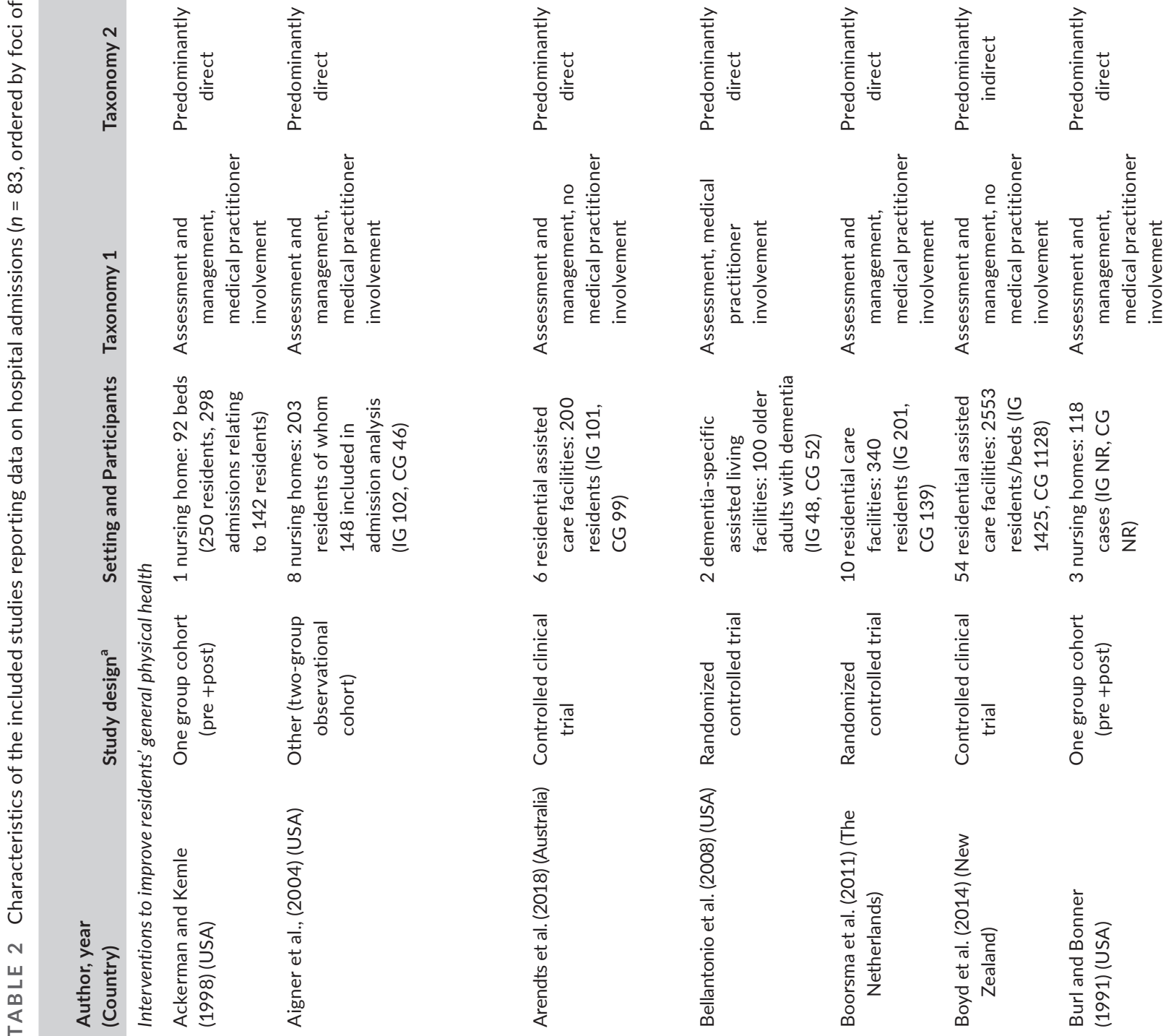

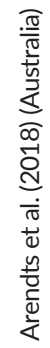

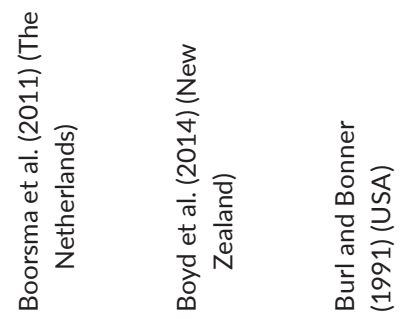




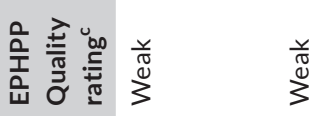

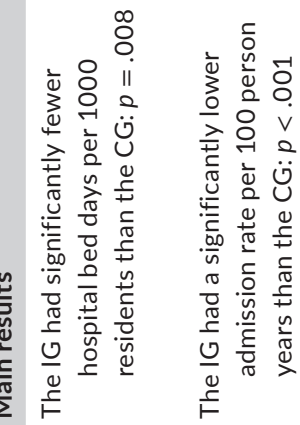

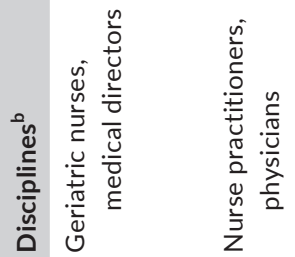

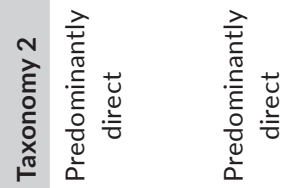

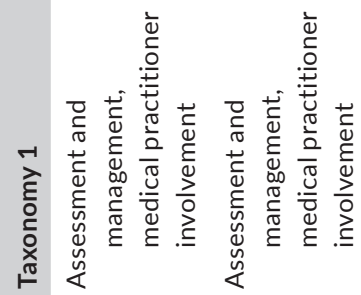

总

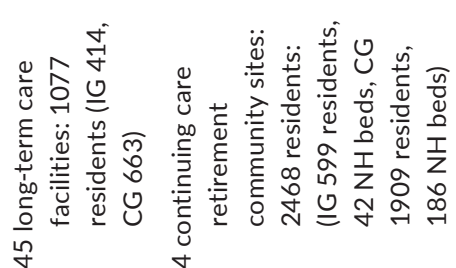

.

N
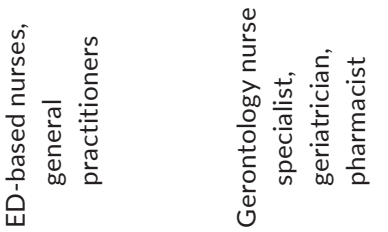

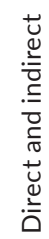

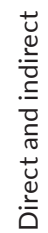

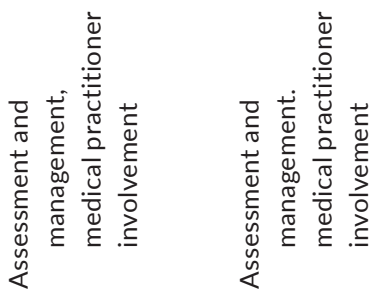

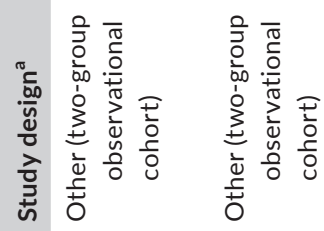

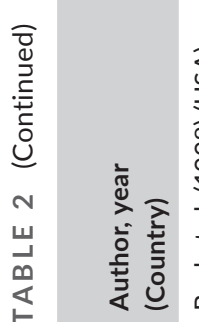

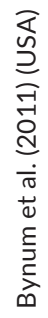

$\frac{0}{50}$
$\frac{5}{50}$
$\frac{5}{2}$

年

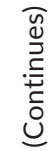
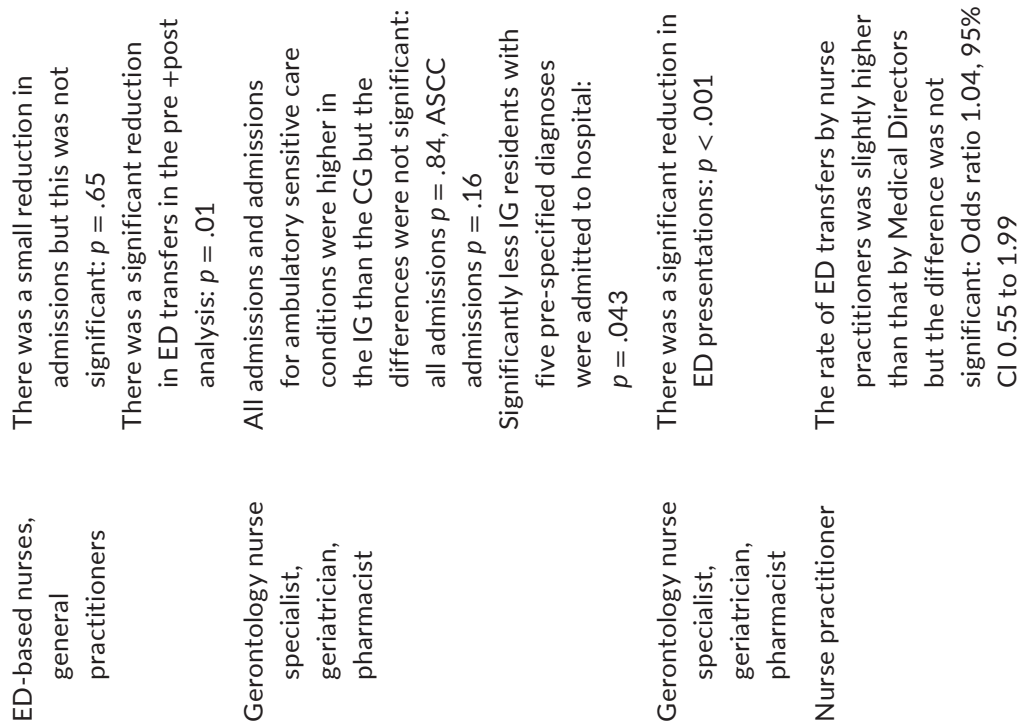

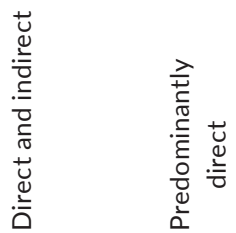

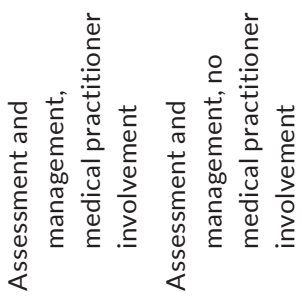

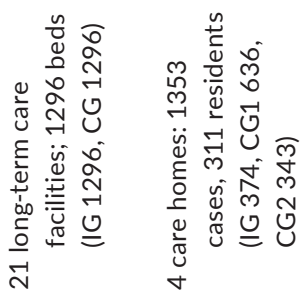
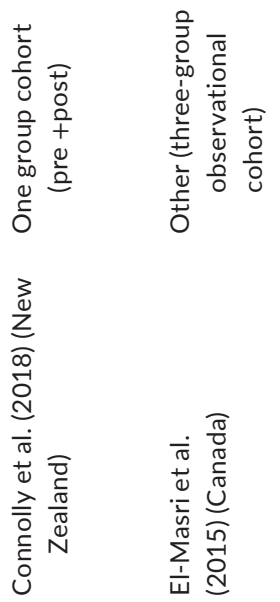

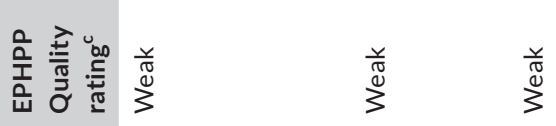

$\sum_{3}^{\frac{2}{\pi}}$

$\frac{0}{\pi}$
$\frac{\pi}{0}$
$\frac{0}{0}$
$\Sigma$

$\stackrel{\frac{1}{\pi}}{3}$

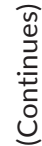

๗ัँ

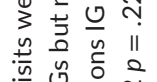

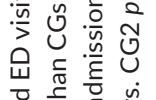

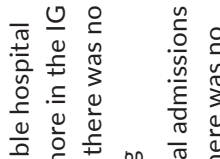

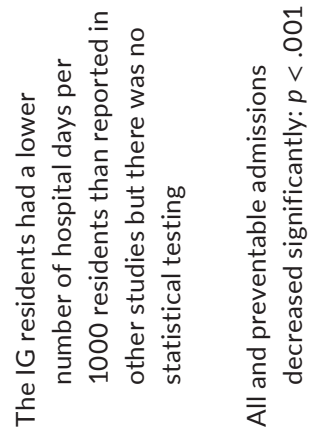

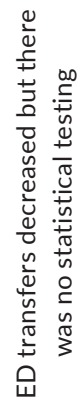

产

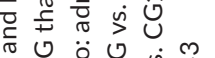

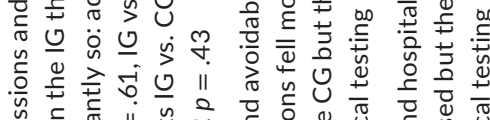

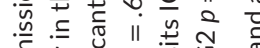

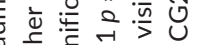

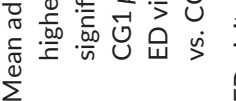

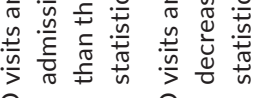

무

离

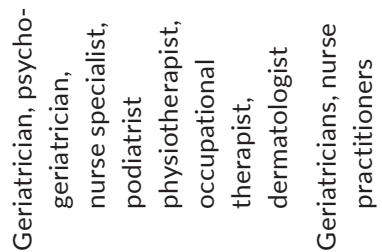

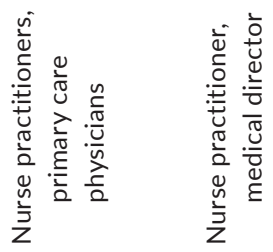

产

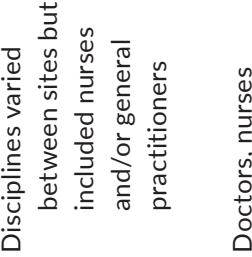

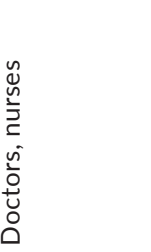

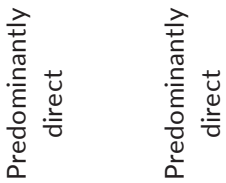

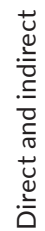

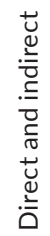

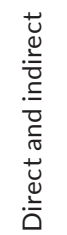
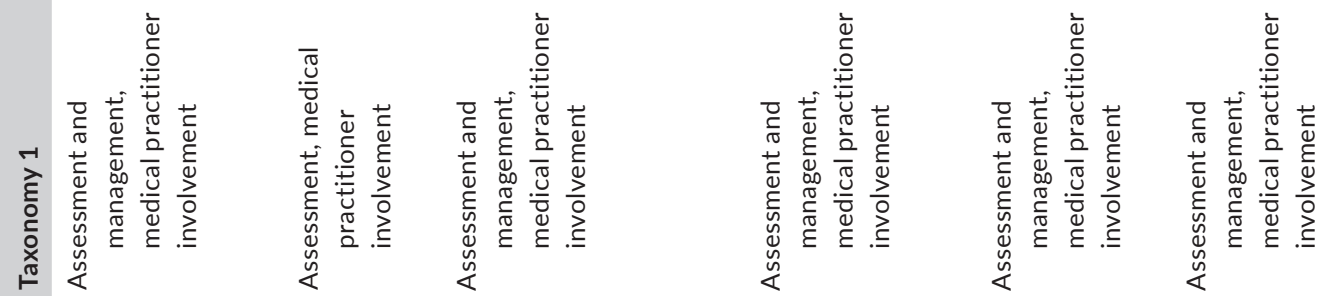

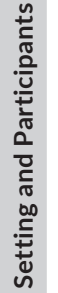

두

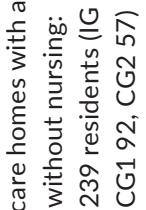

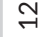

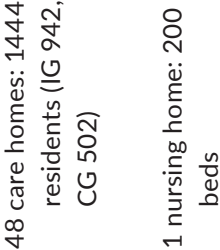

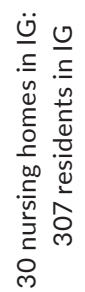

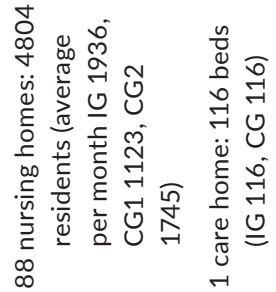

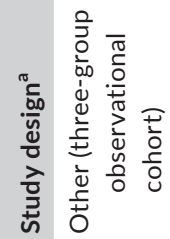

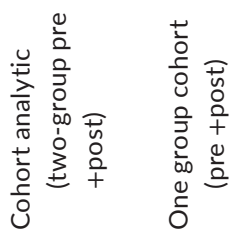

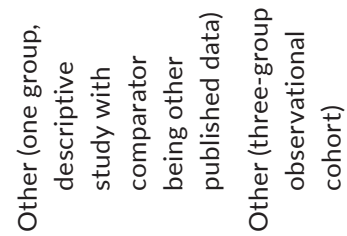

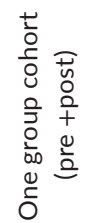
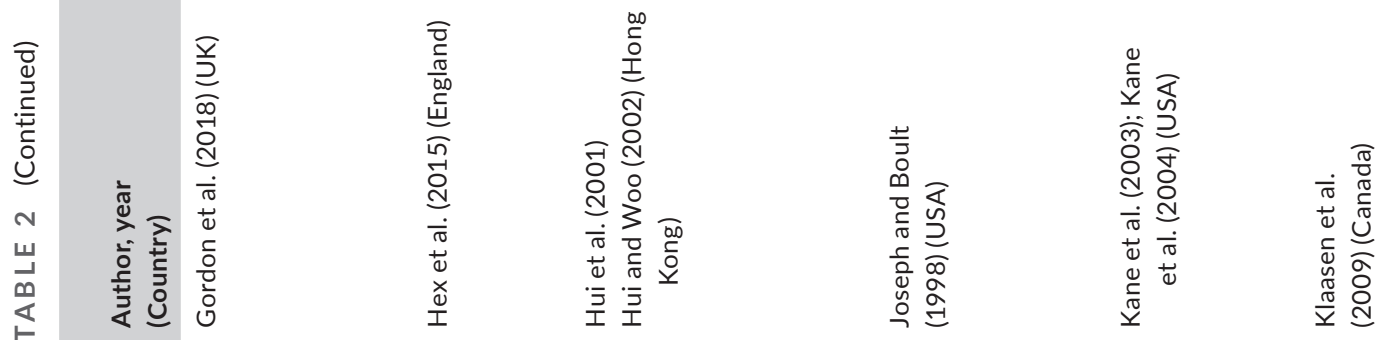


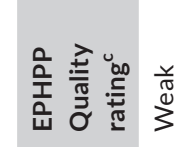

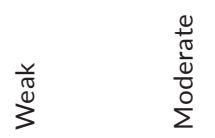

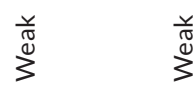

$\stackrel{\text { j }}{3}^{\frac{1}{\pi}}$

$\widetilde{d}$
$\stackrel{\underline{\underline{c}}}{\overline{0}}$
$\underline{0}$
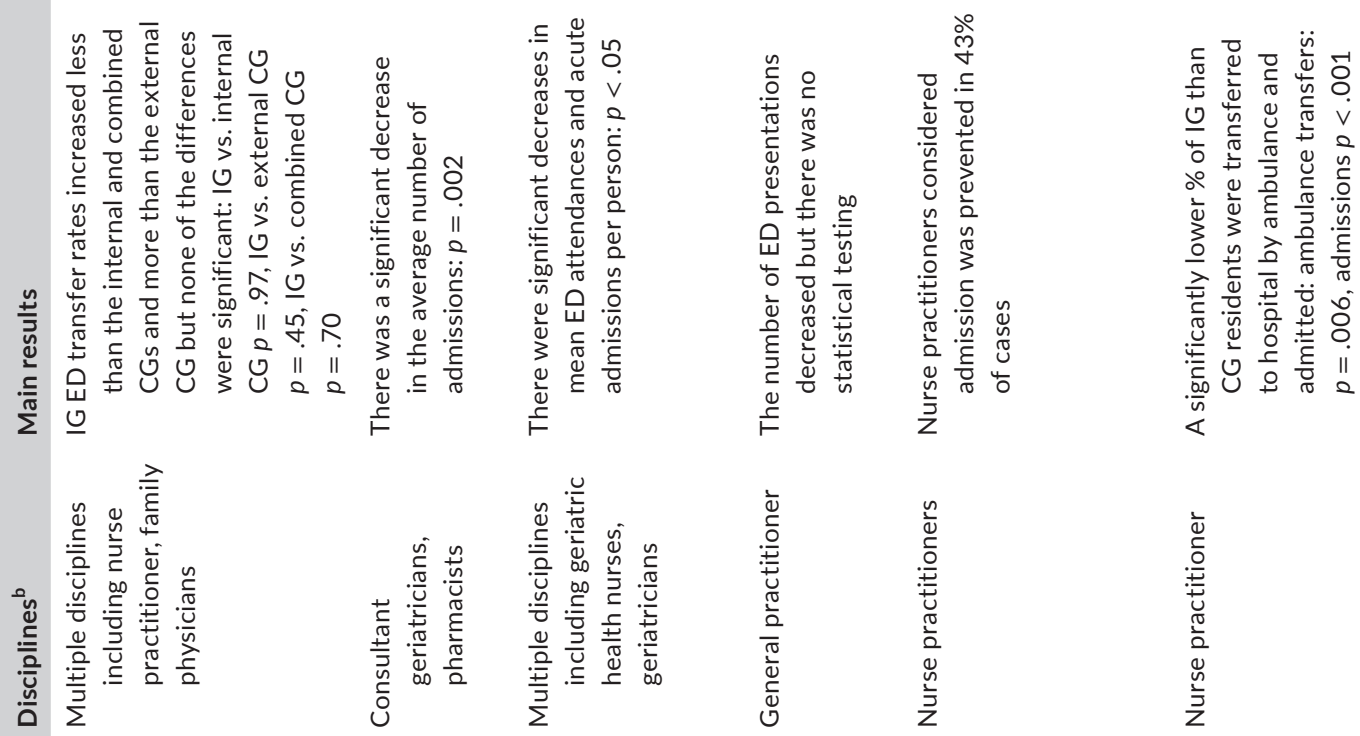

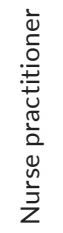
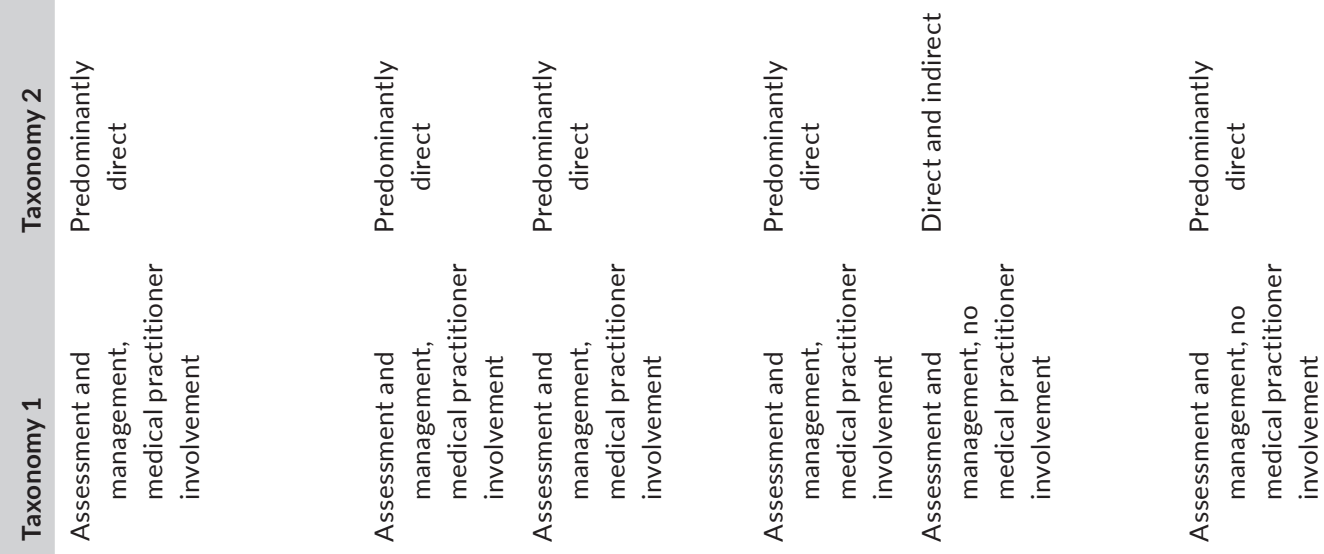

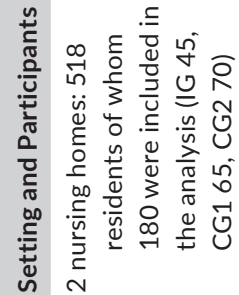
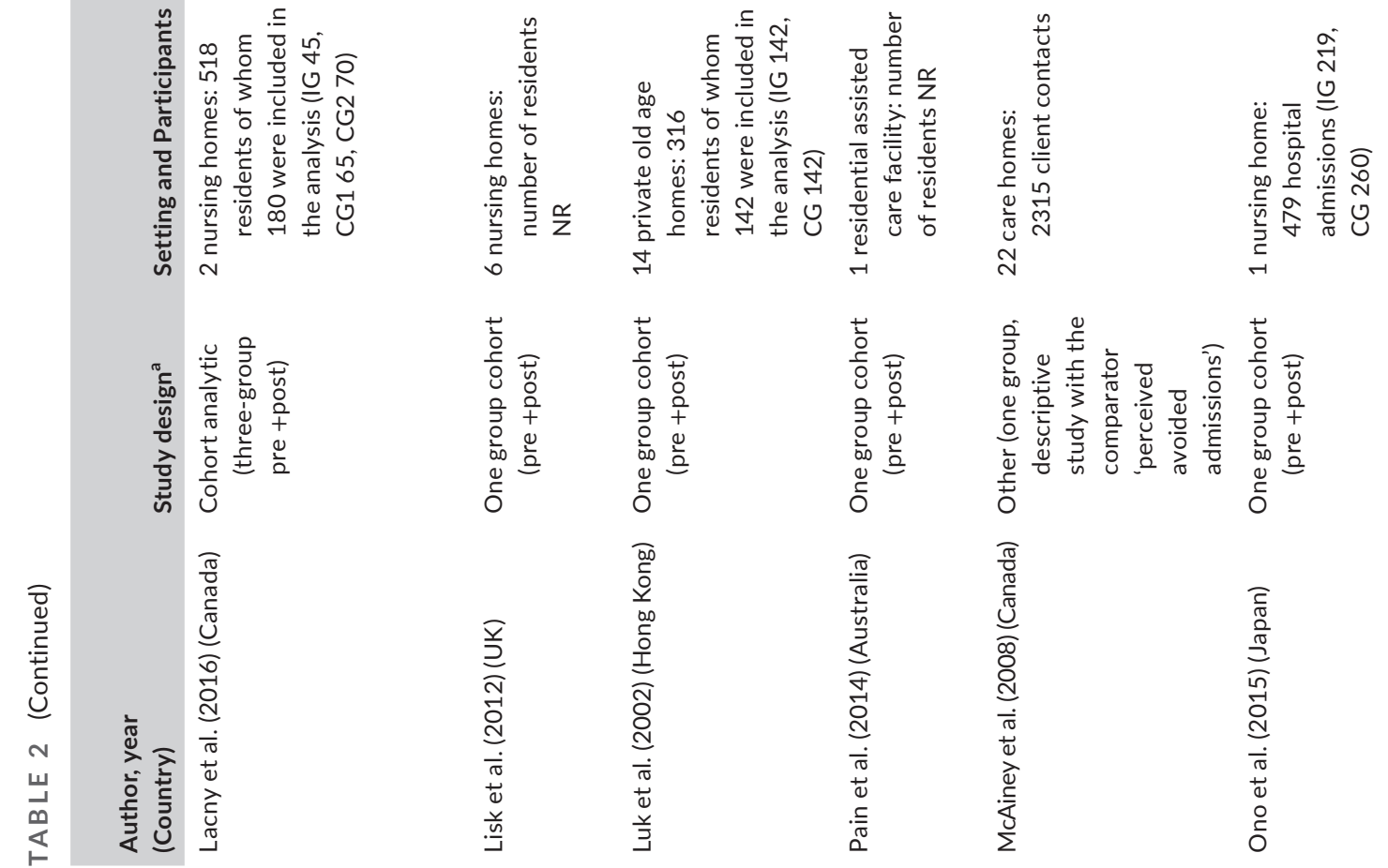


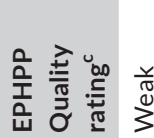

$\frac{0}{\pi}$
$\frac{\pi}{0}$
$\frac{0}{0}$
$\Sigma$

芩

$\stackrel{\frac{10}{3}}{3}$

$\frac{0}{0}$
$\frac{\pi}{0}$
$\frac{0}{2}$
$\Sigma$

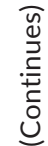
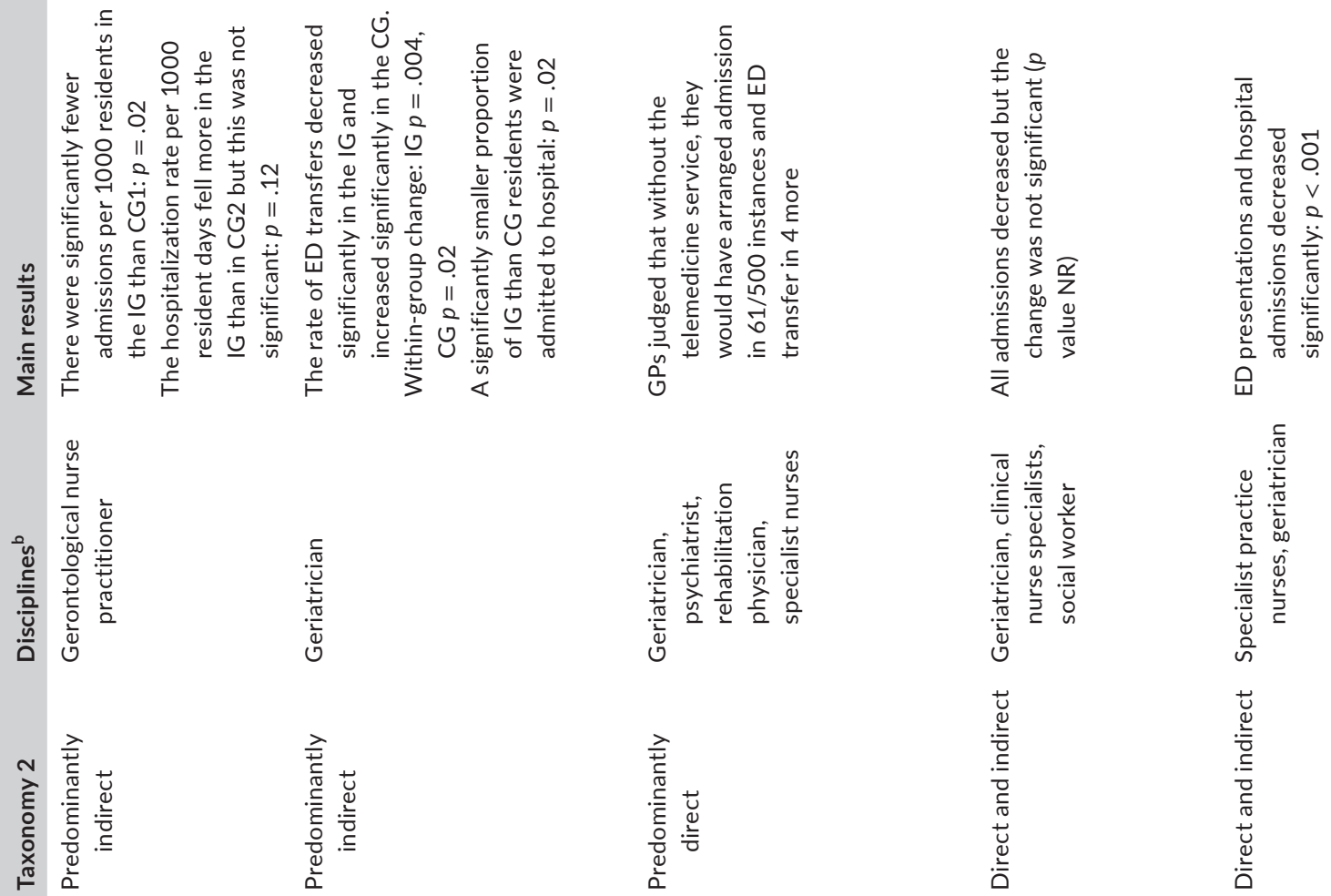

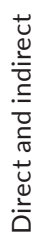

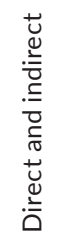
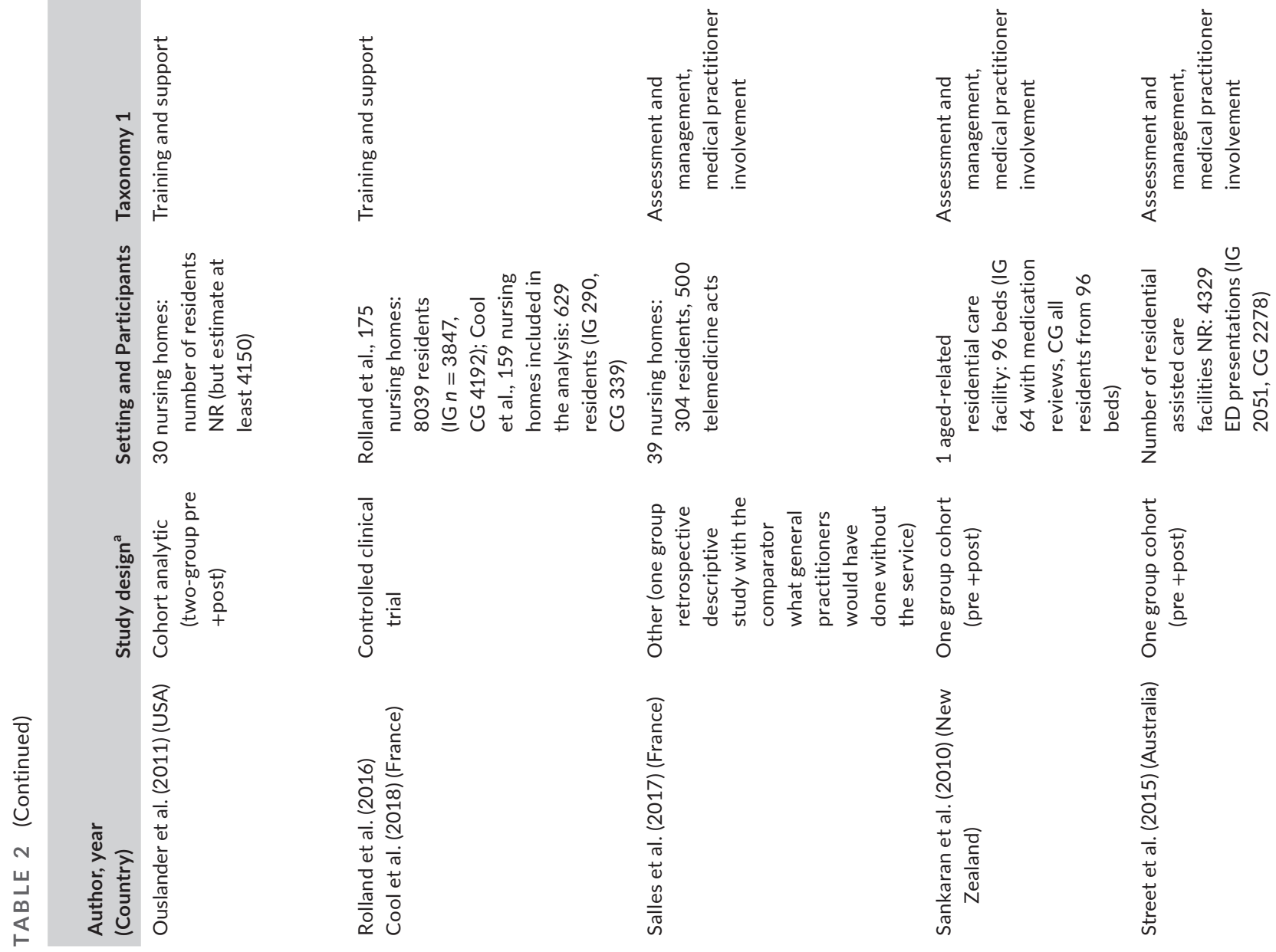

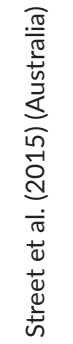




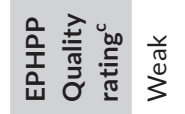

I

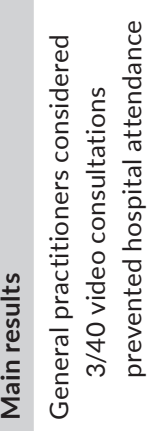

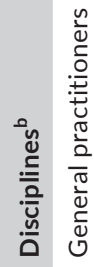

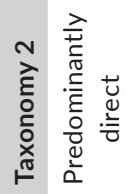

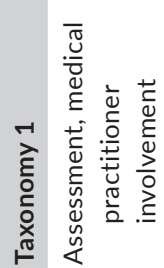

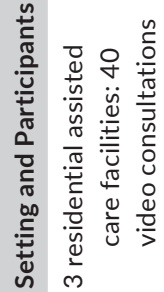

$\frac{0}{\pi}$
$\frac{\pi}{0}$
$\frac{0}{0}$
$\Sigma$

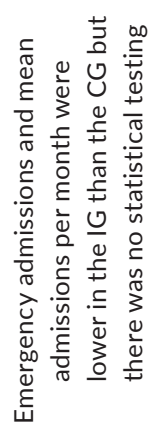

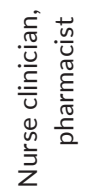

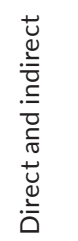

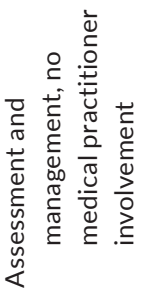

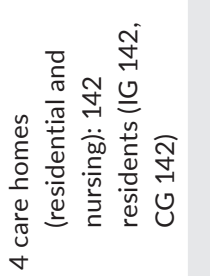

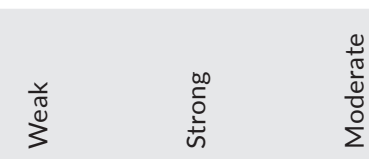

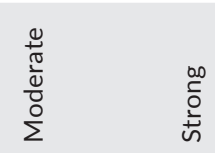

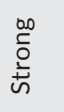

光

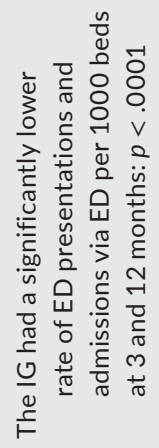

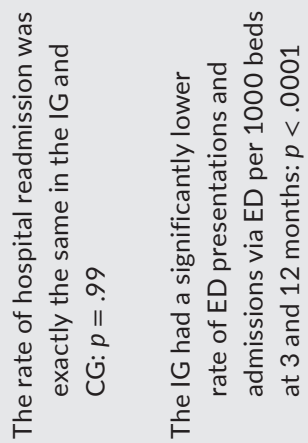
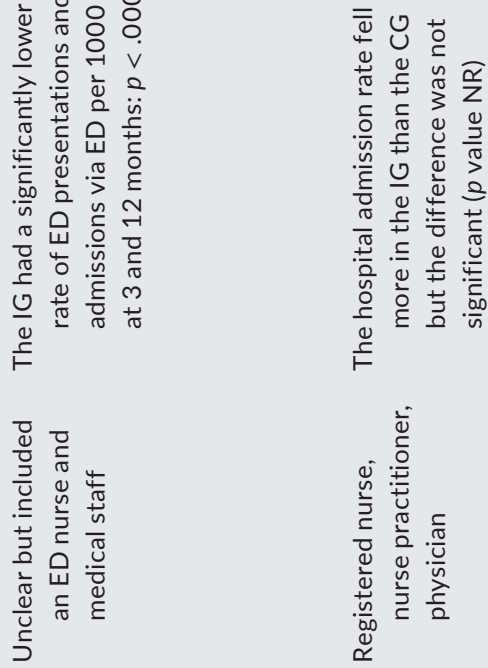

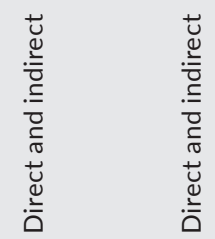

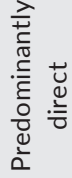

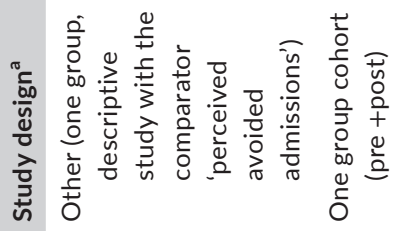

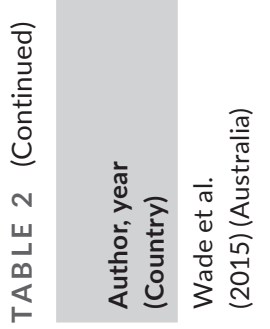

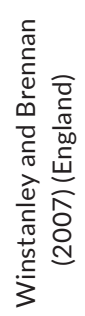

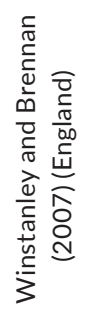

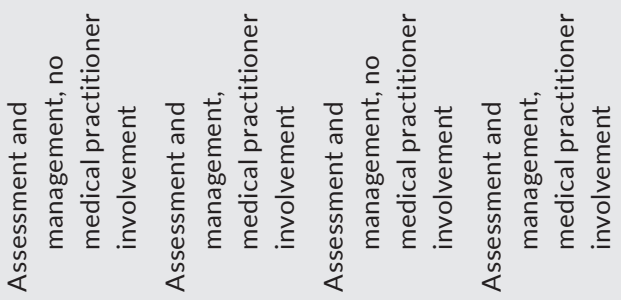

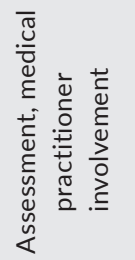

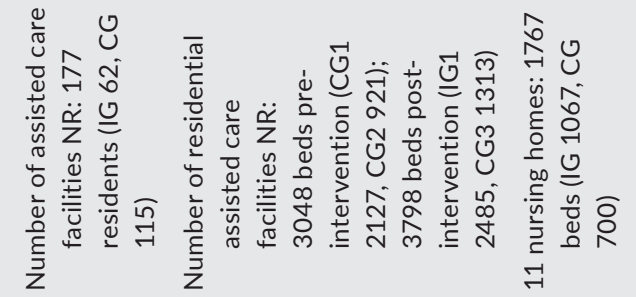

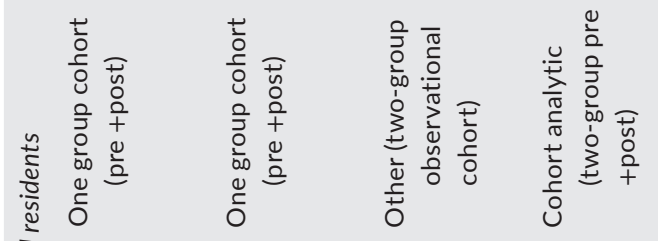

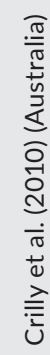

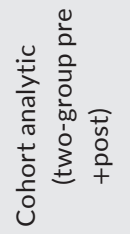

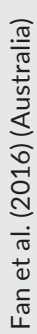

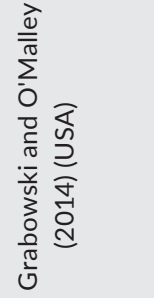



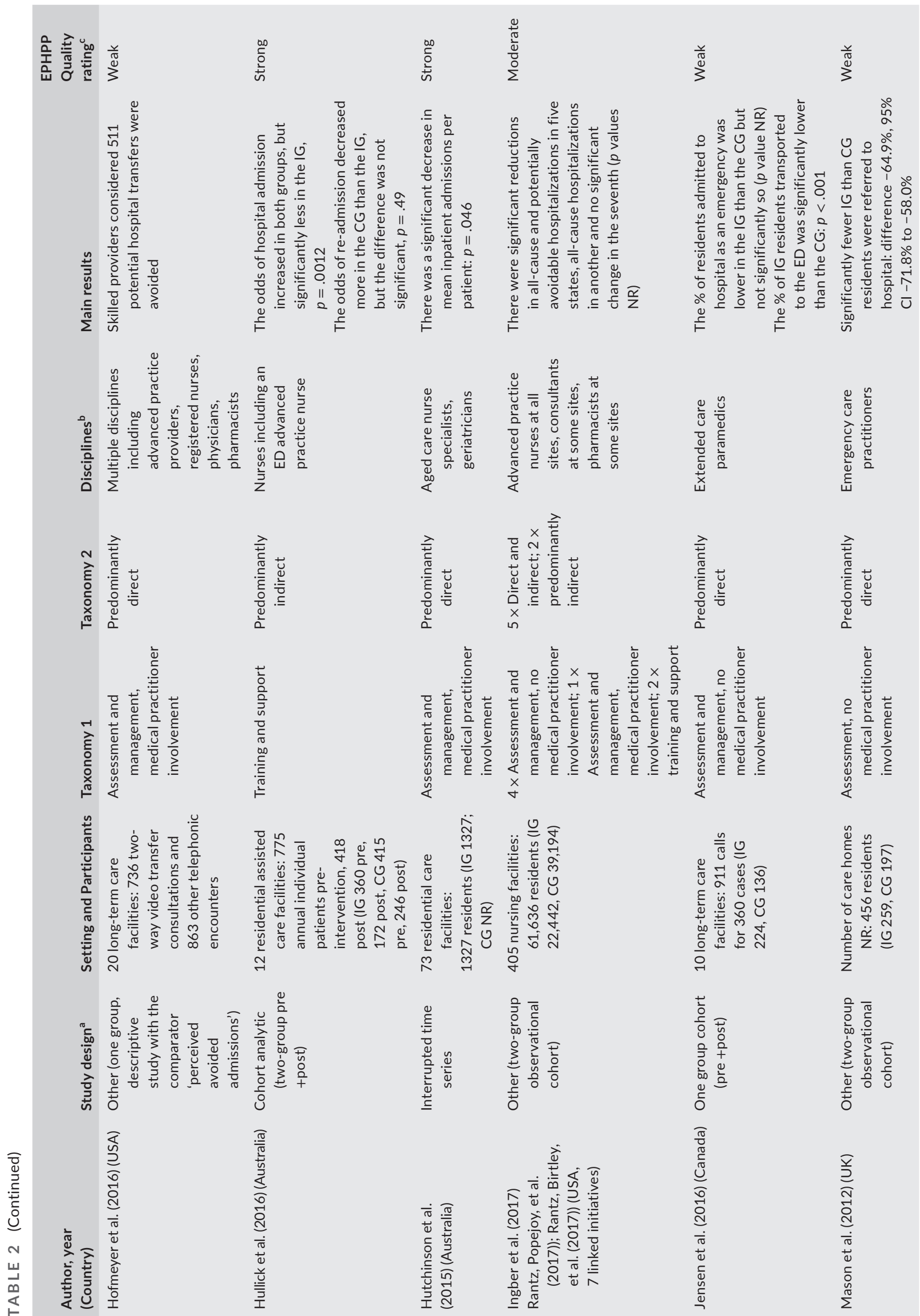


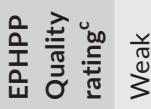

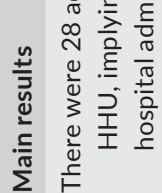

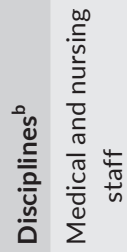

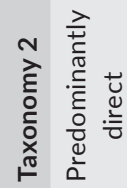
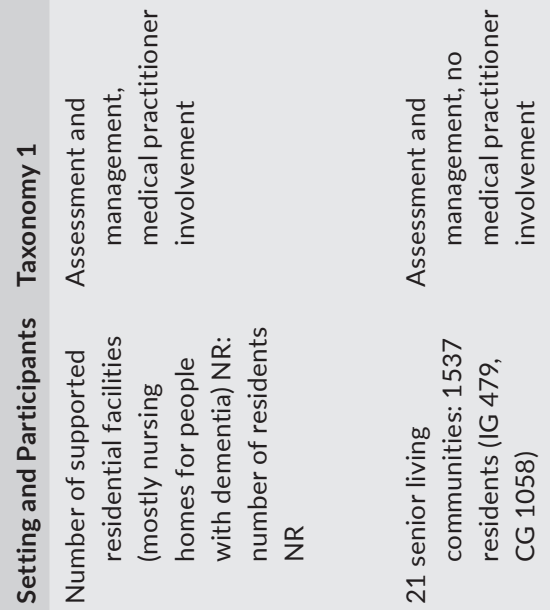

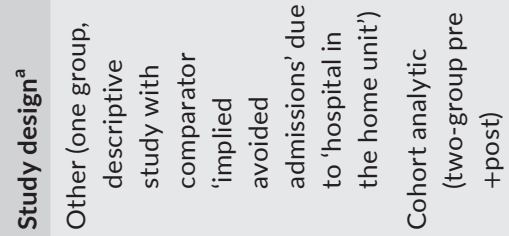

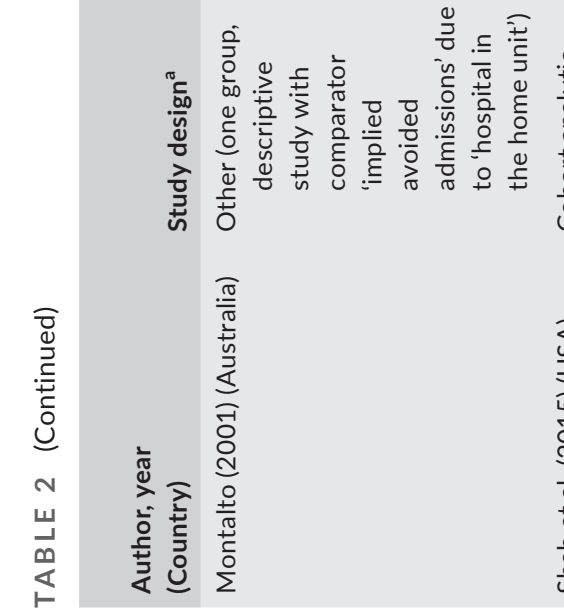

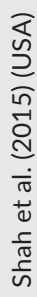

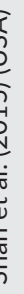

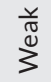

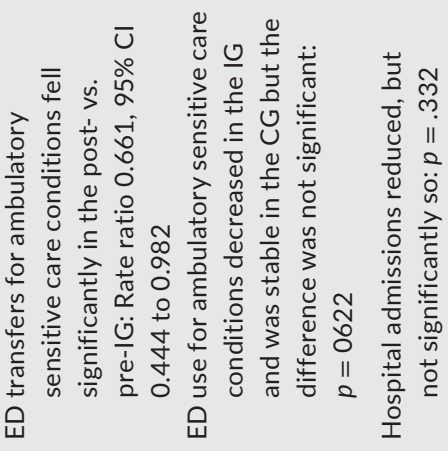

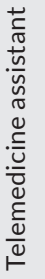

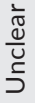

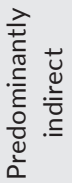

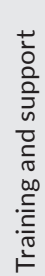

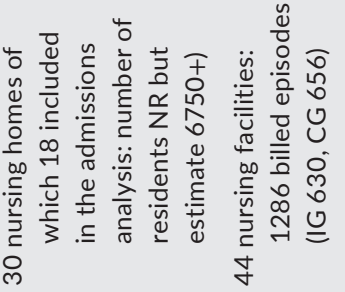

$\stackrel{\frac{1}{\pi}}{3}$

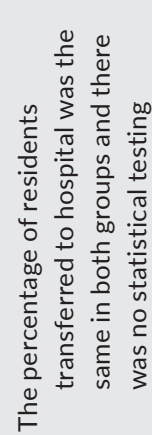

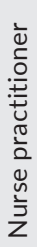

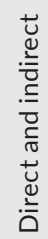

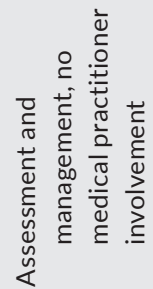

$\frac{\stackrel{0}{\pi}}{\frac{0}{0}}$
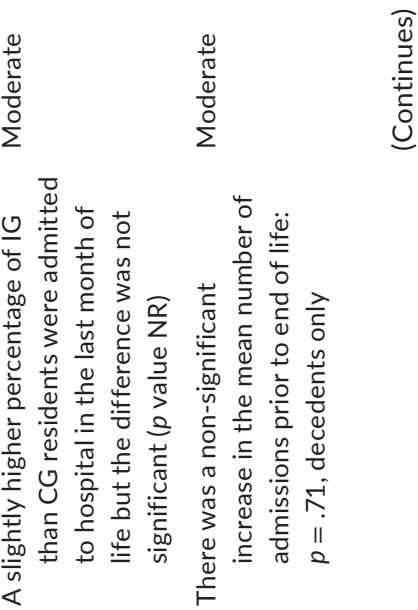

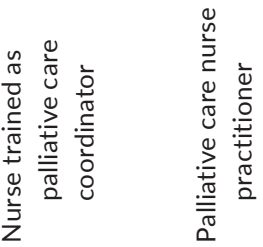
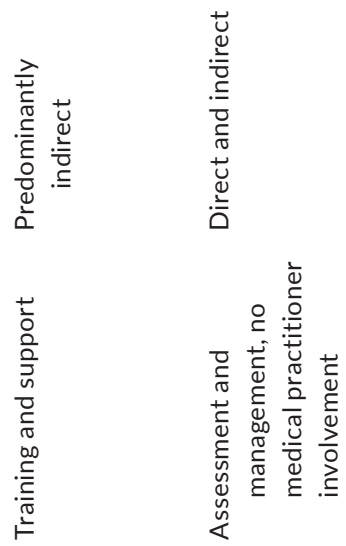
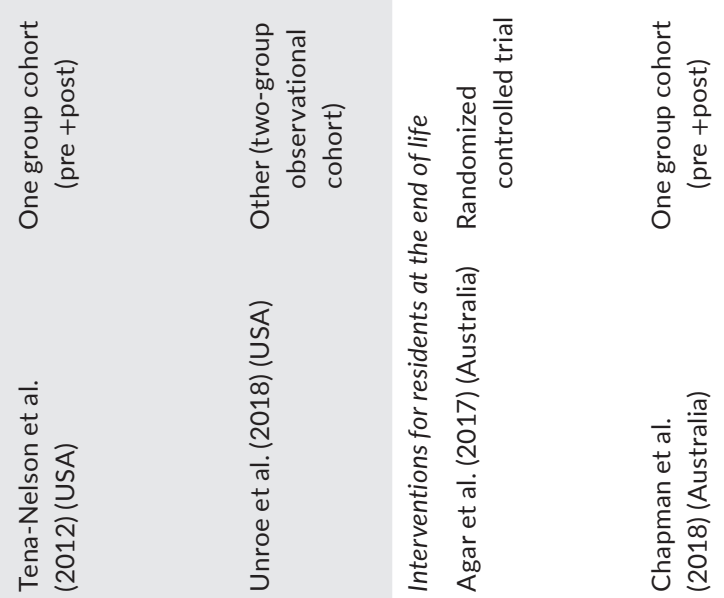

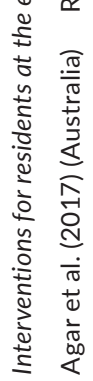

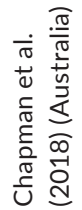




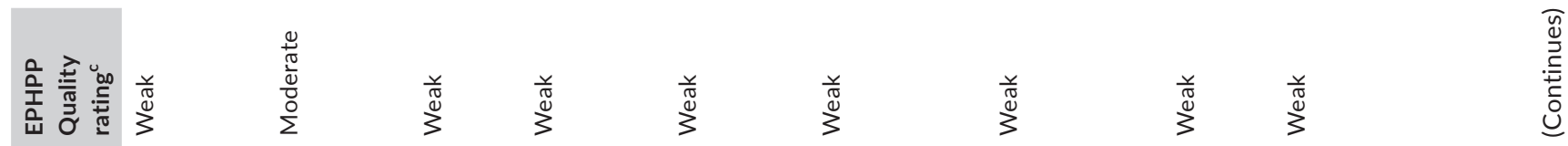

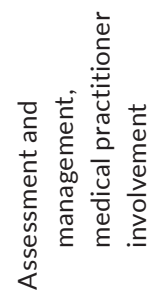

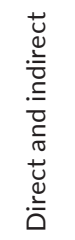
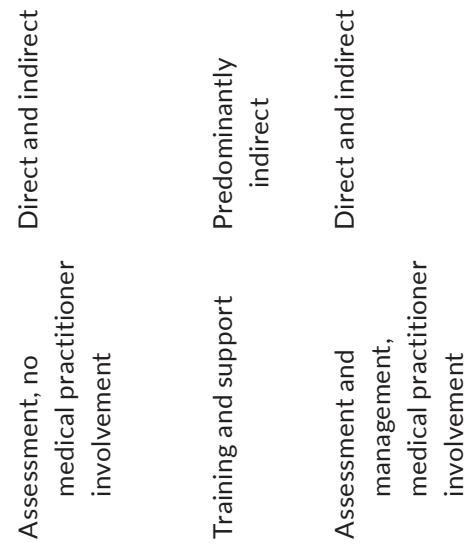

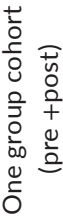

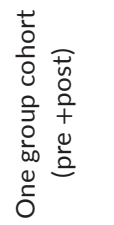

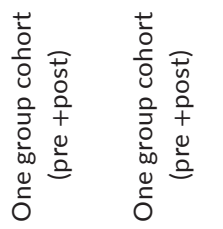

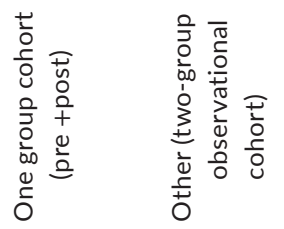

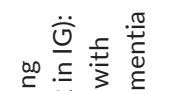

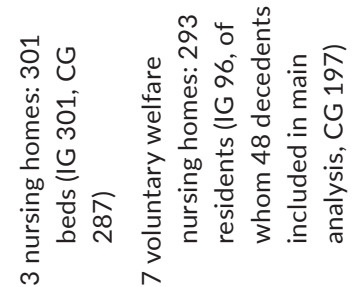
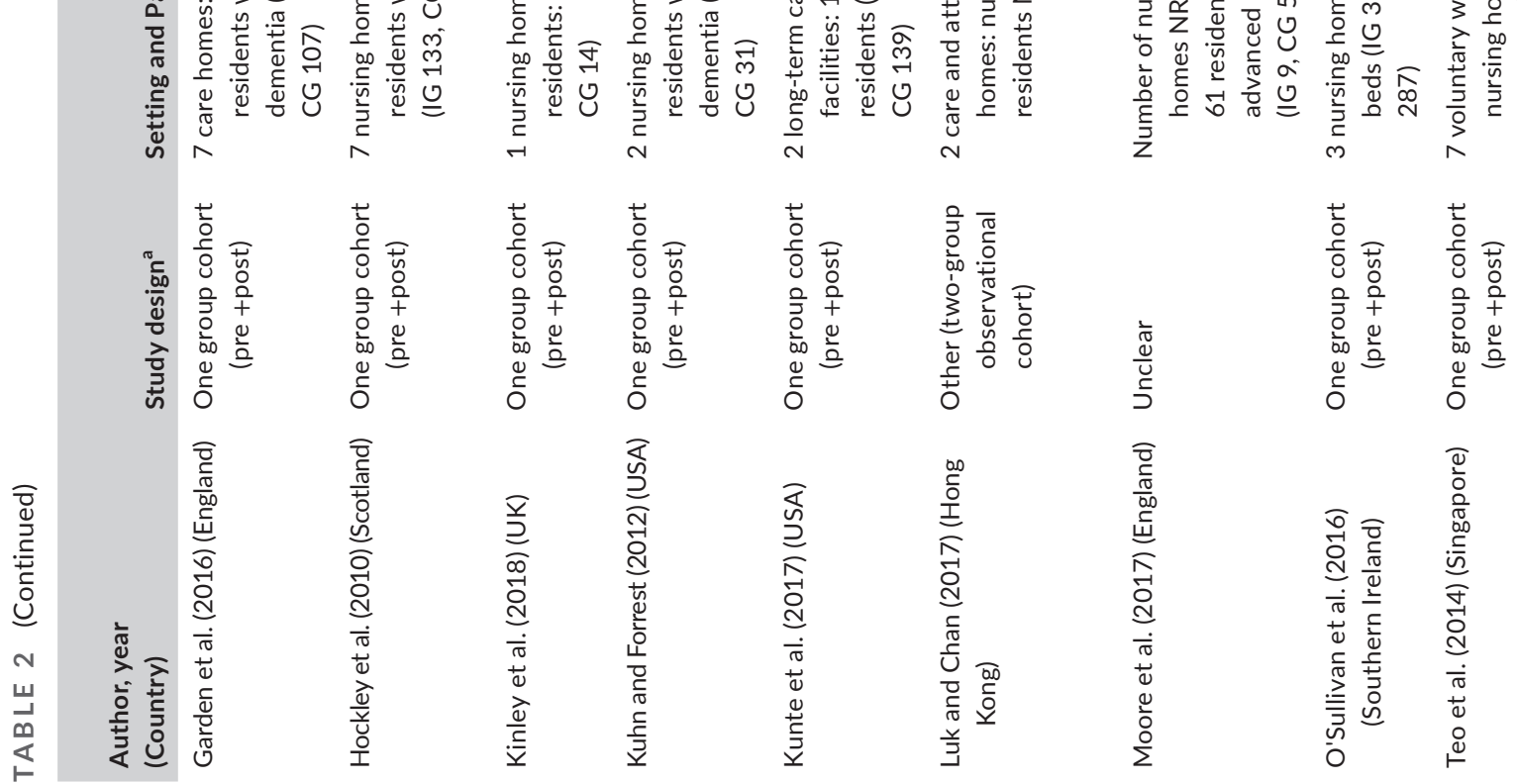


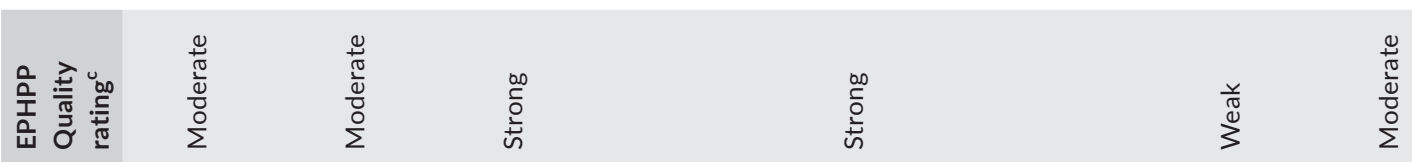

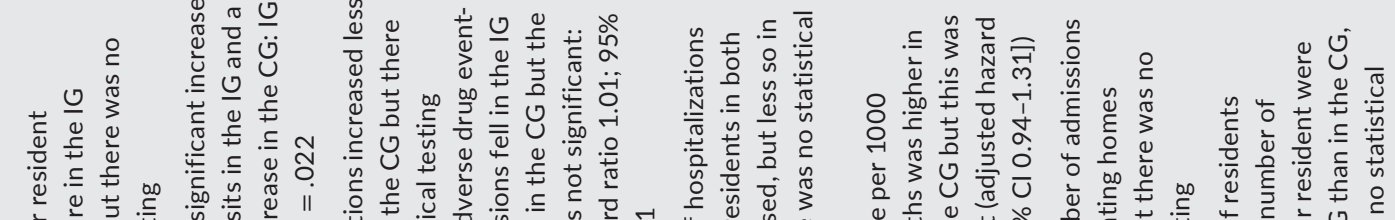

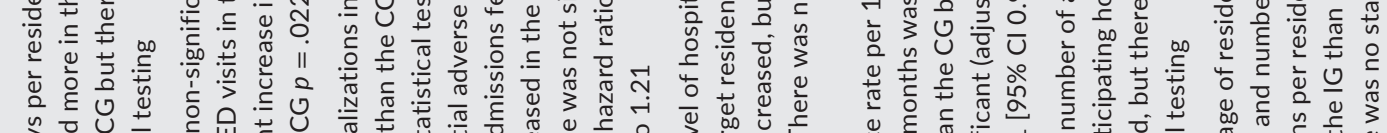

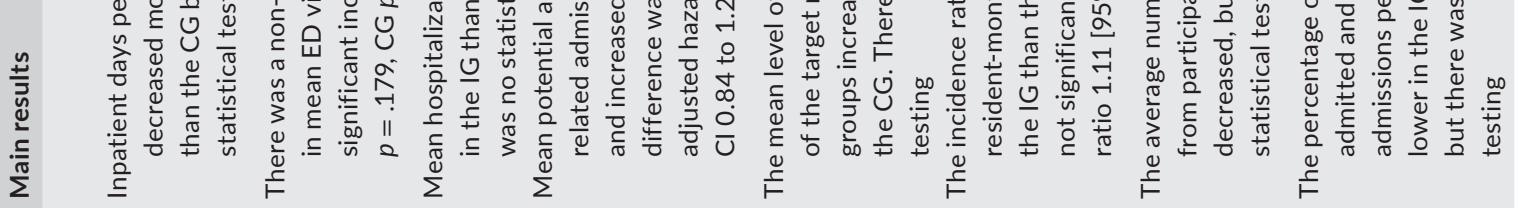

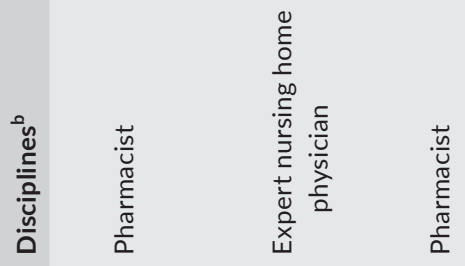

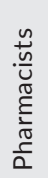

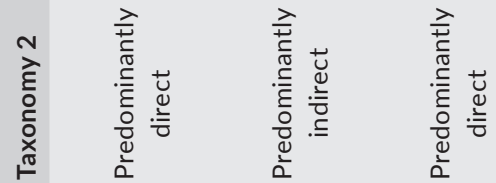

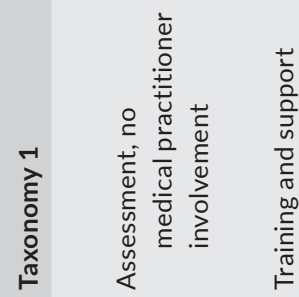

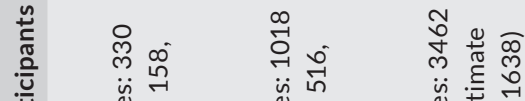

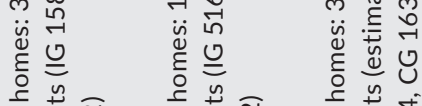

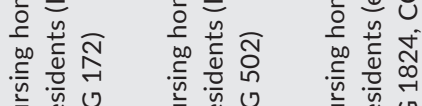

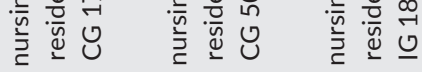
$\checkmark$

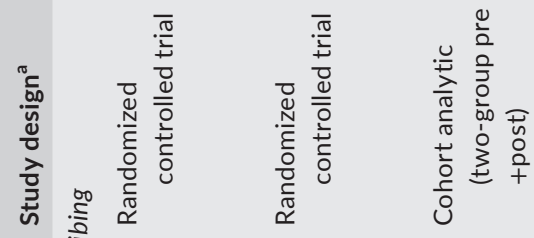

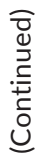

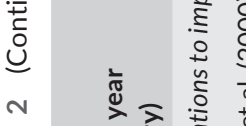

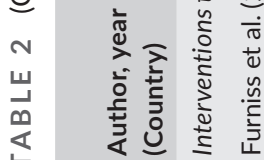

ले

พ

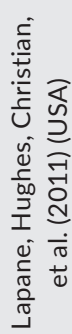

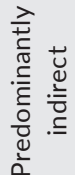

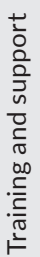

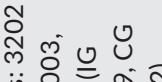

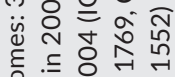

of

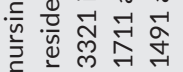

光

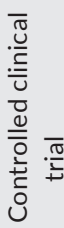

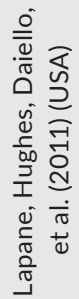

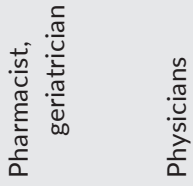

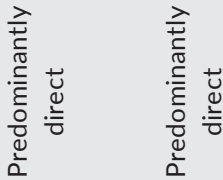

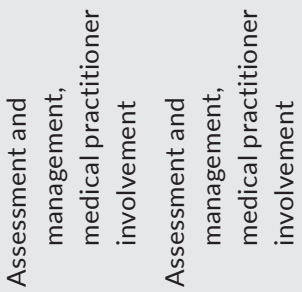

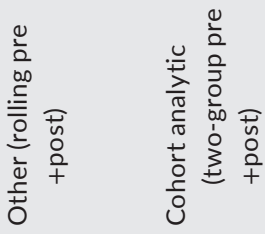

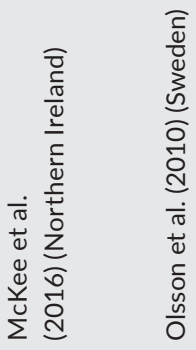



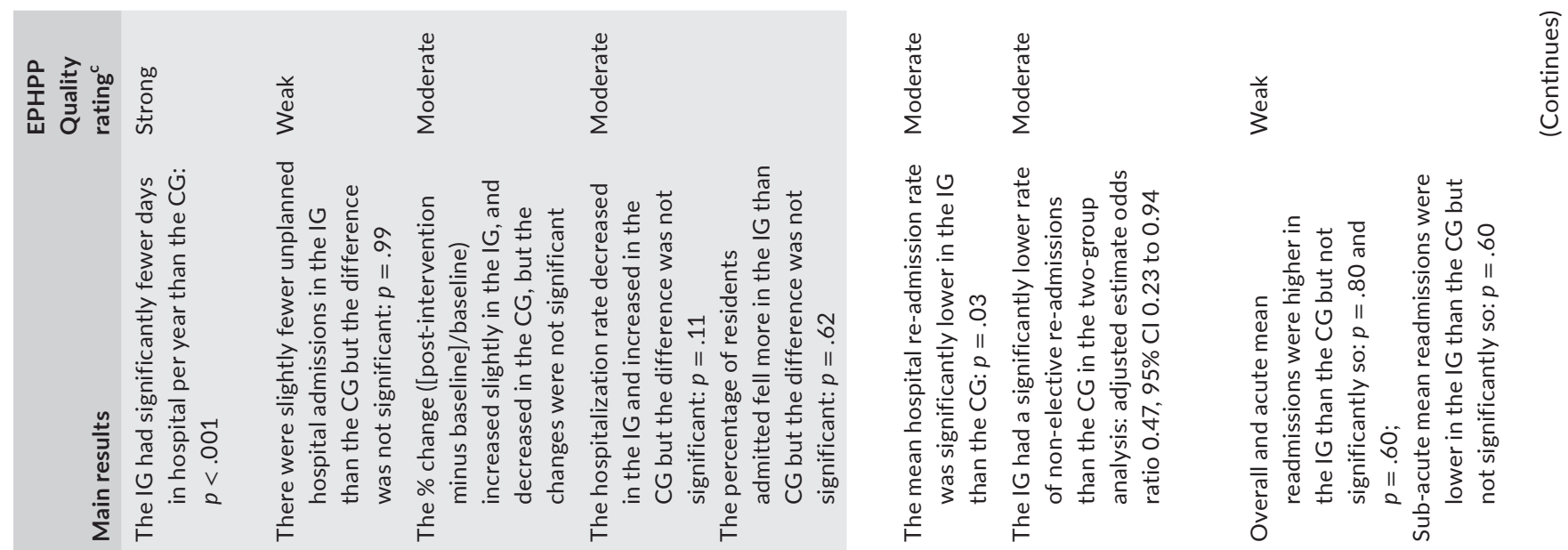

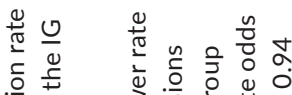

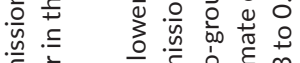

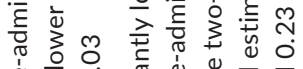

过文

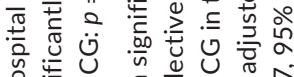

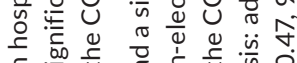

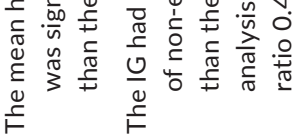
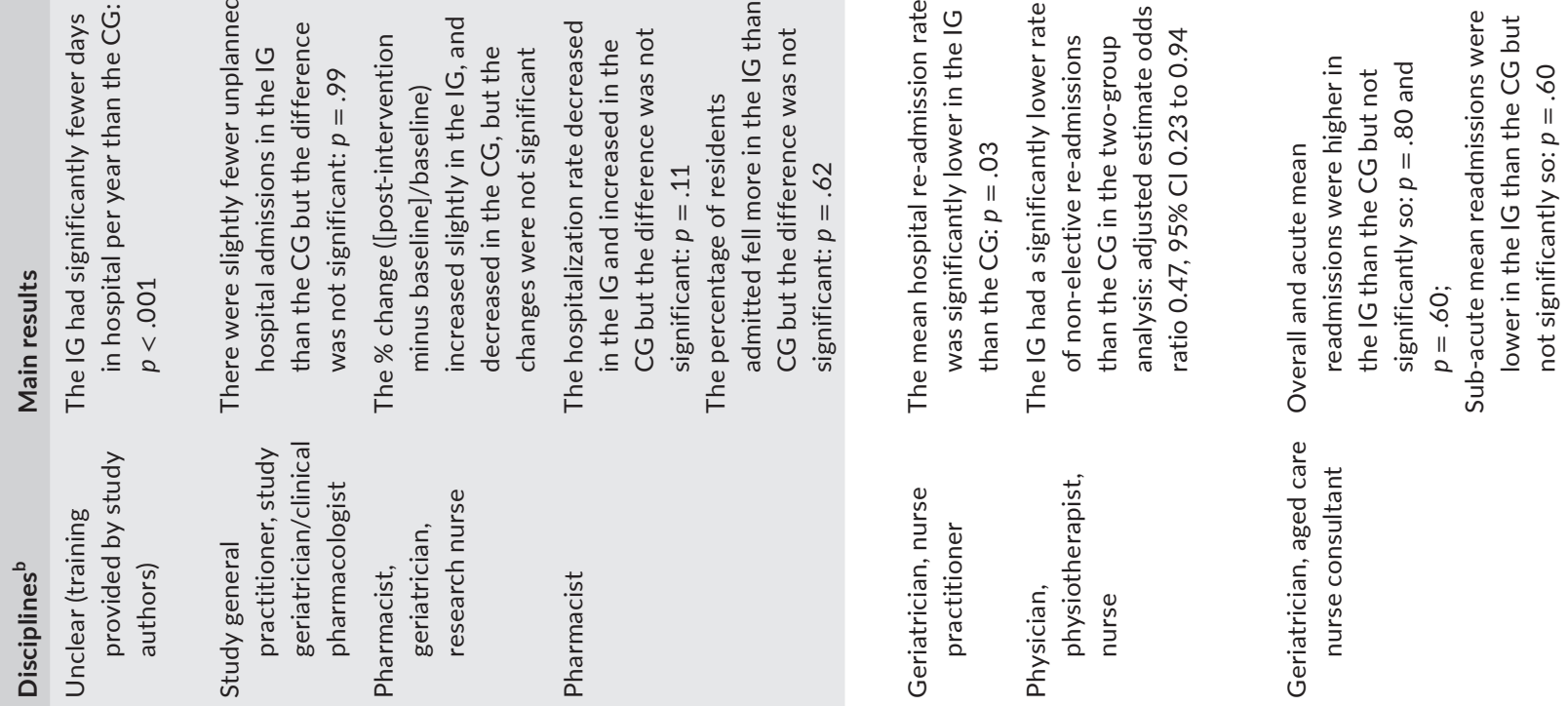

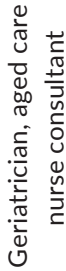
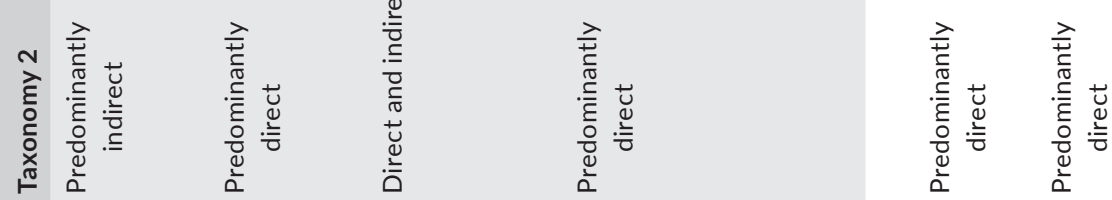

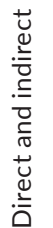
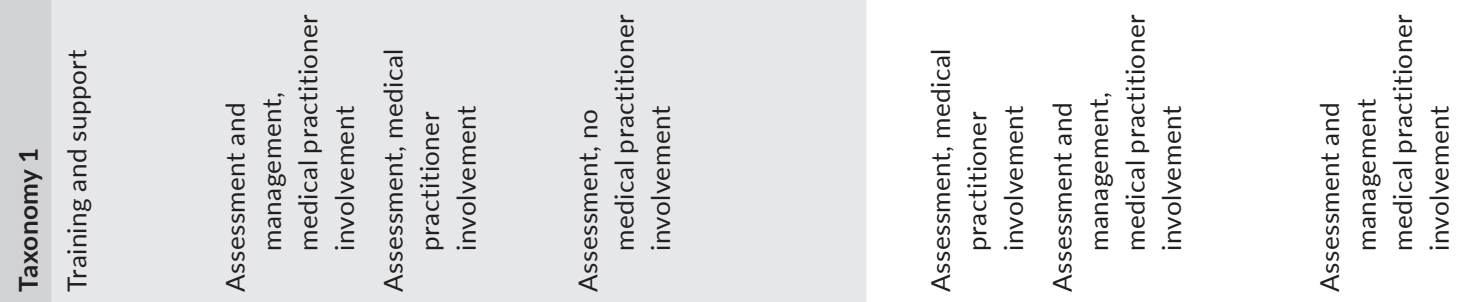

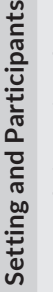

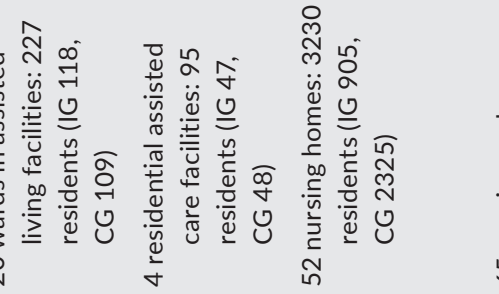

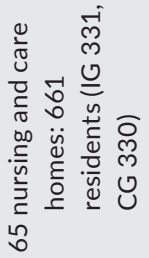

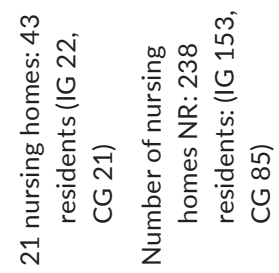
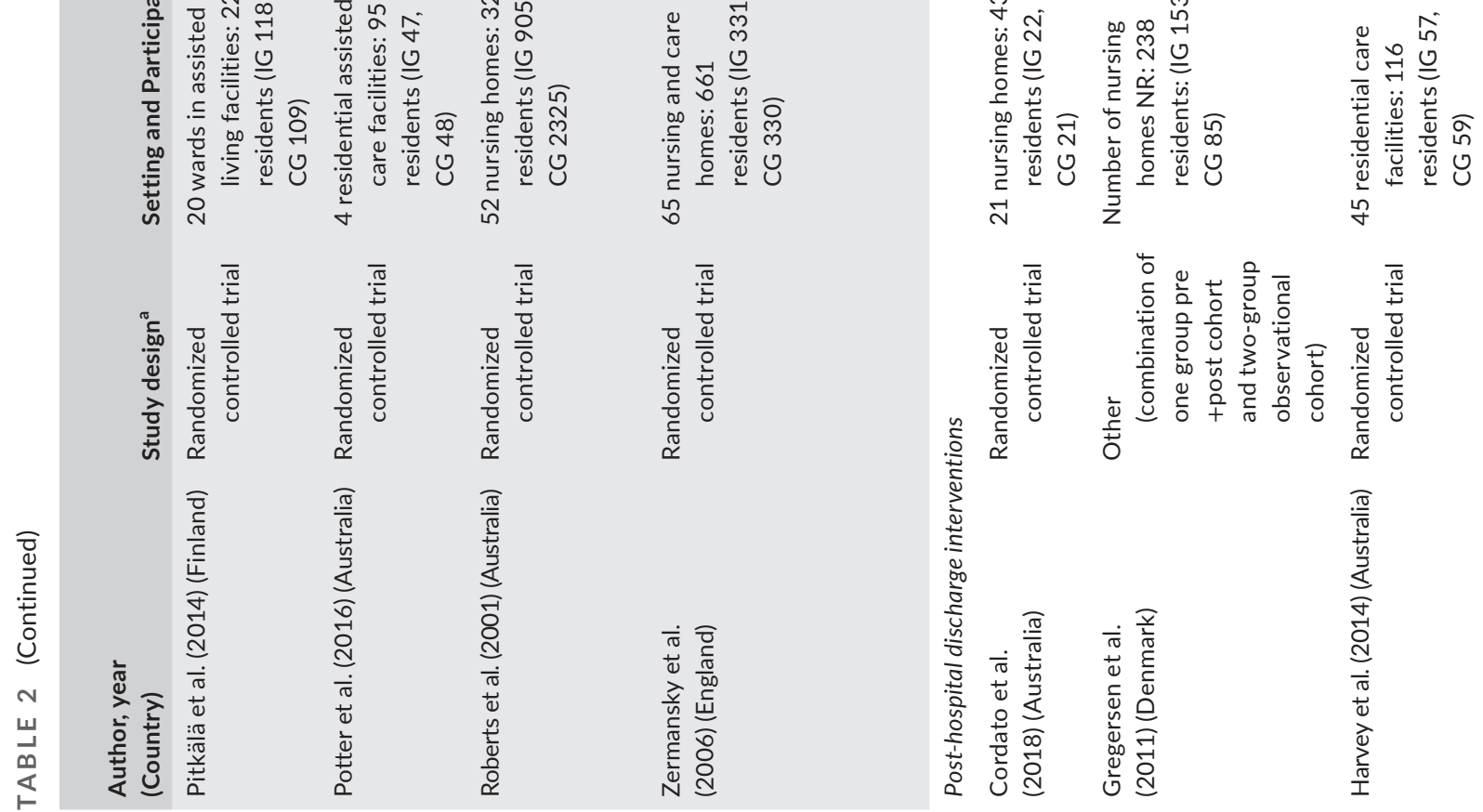


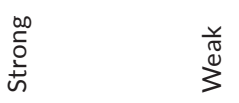

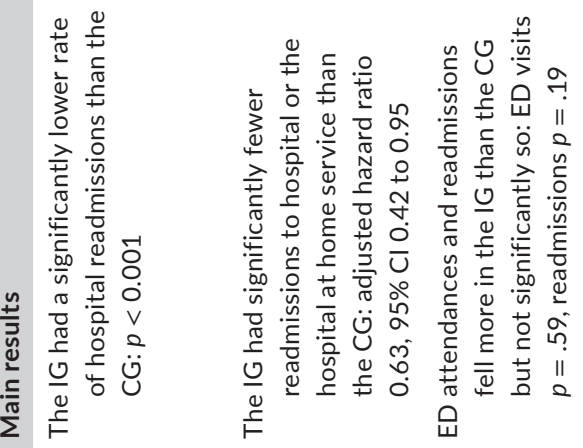

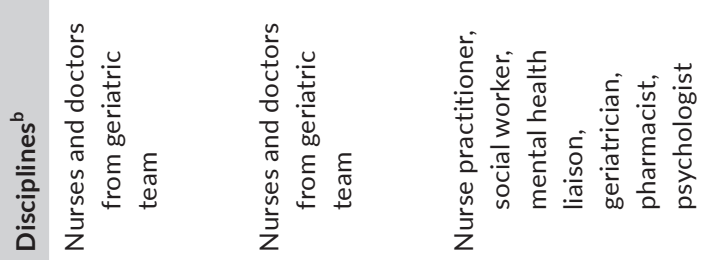

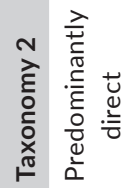

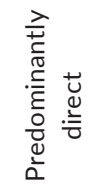
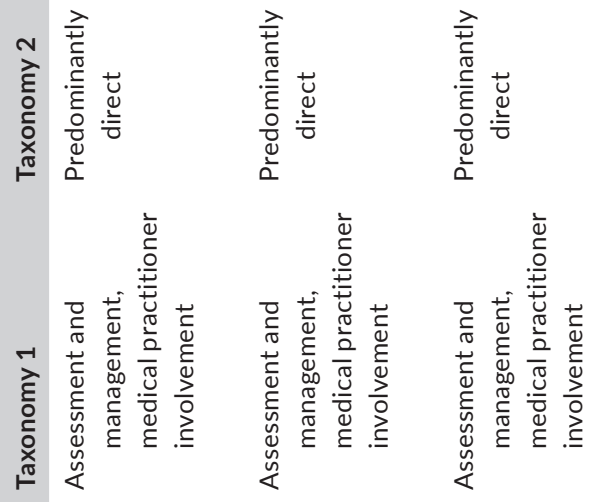

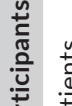

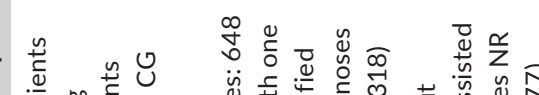

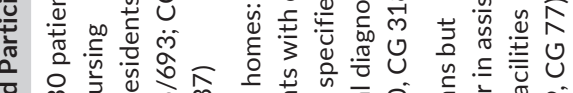

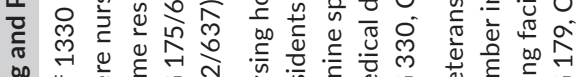

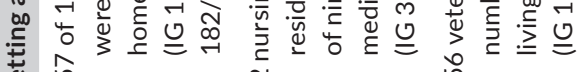
ज

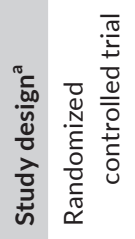

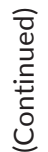
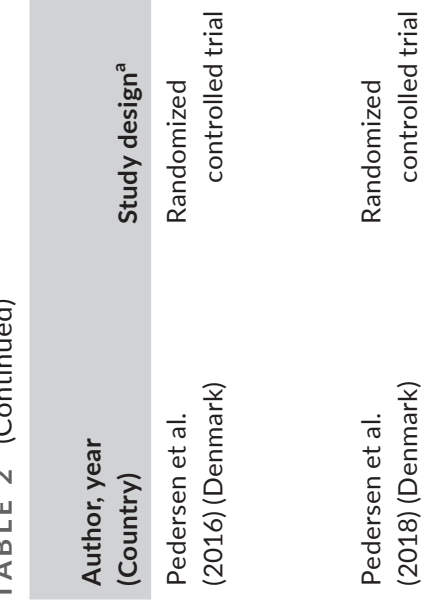

$\frac{0}{\frac{\pi}{\pi}}$
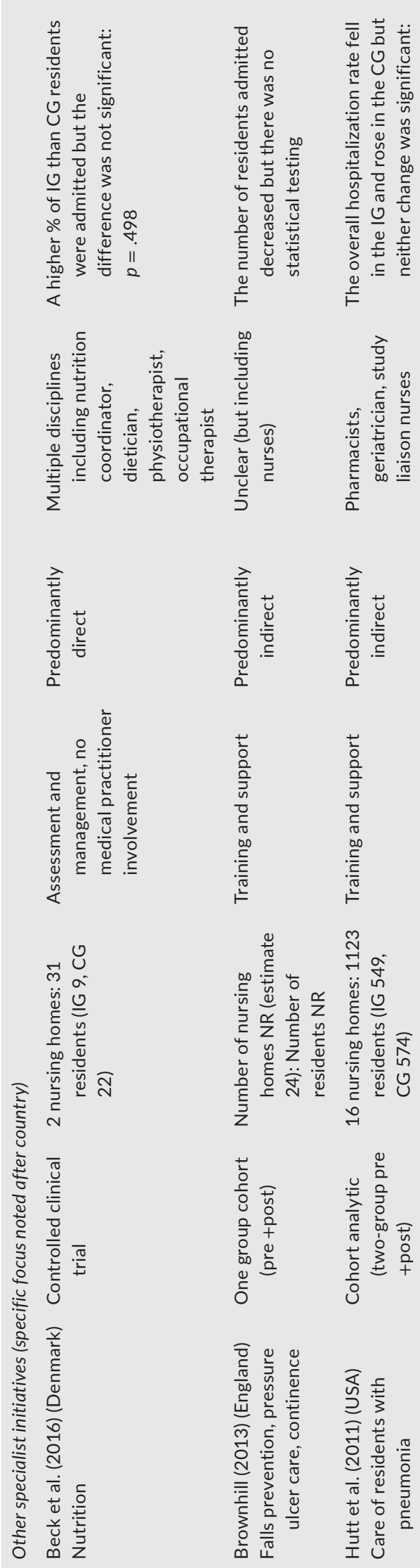


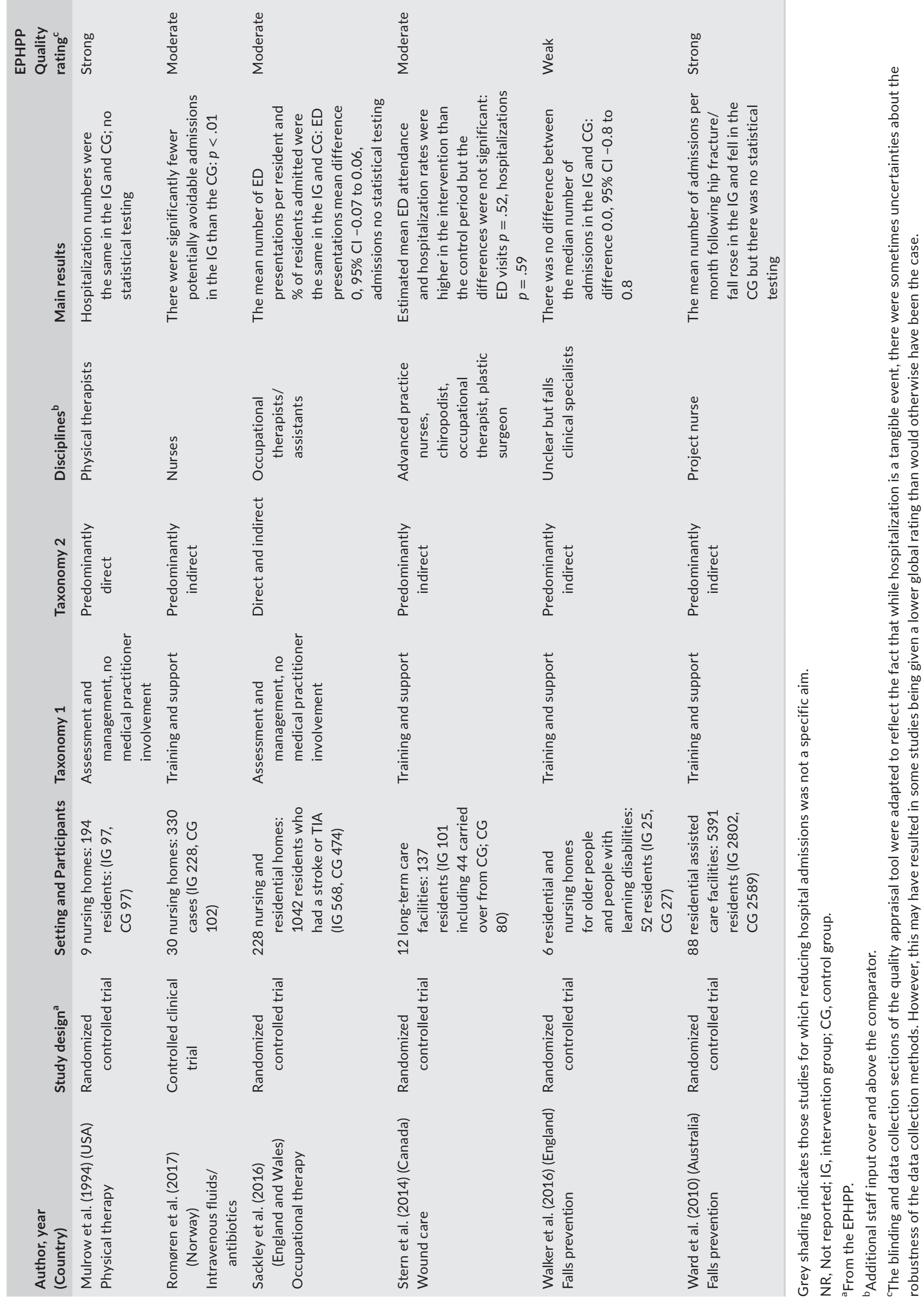



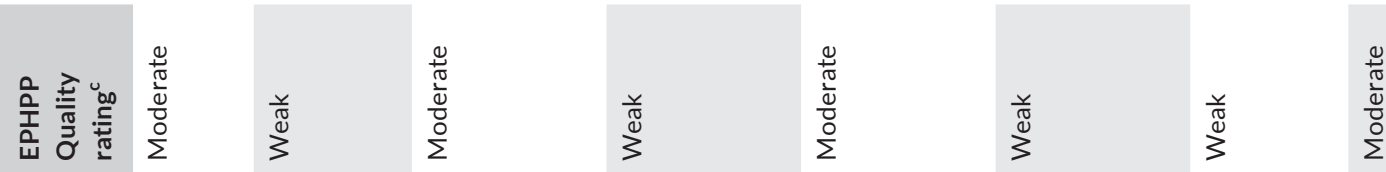

InWm
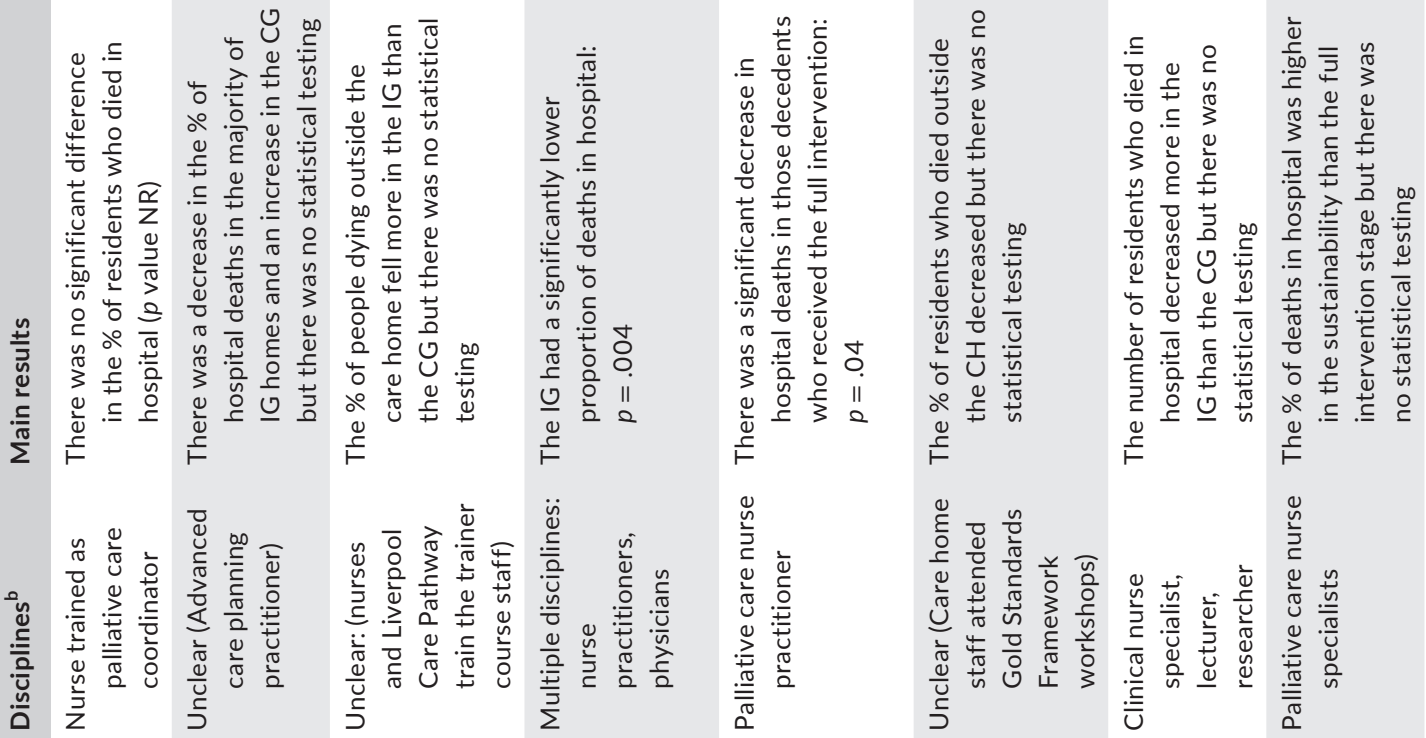

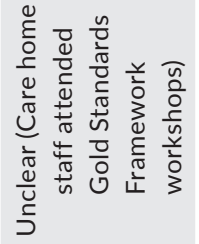

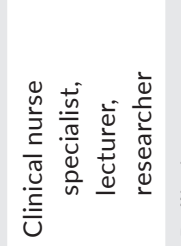

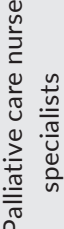
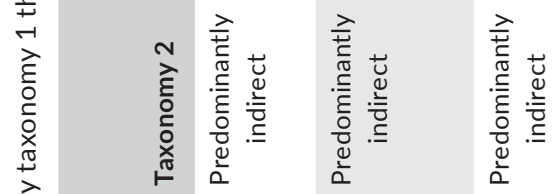

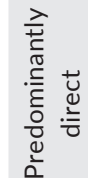
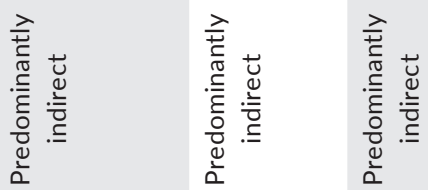

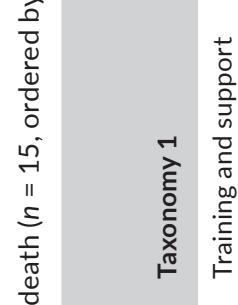
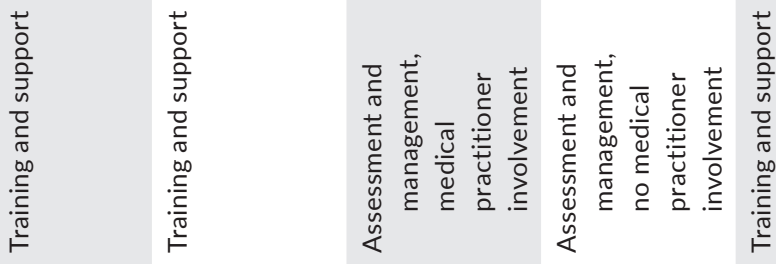

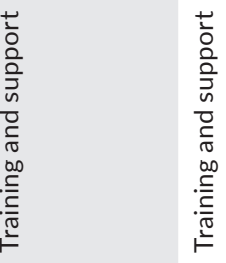

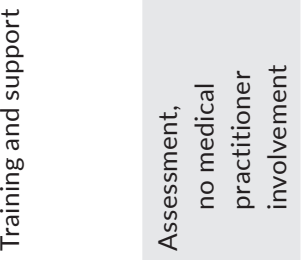

4
$\stackrel{0}{0}$
$\frac{\pi}{2}$
$\frac{\pi}{2}$

$\frac{\pi}{\frac{\pi}{0}}$

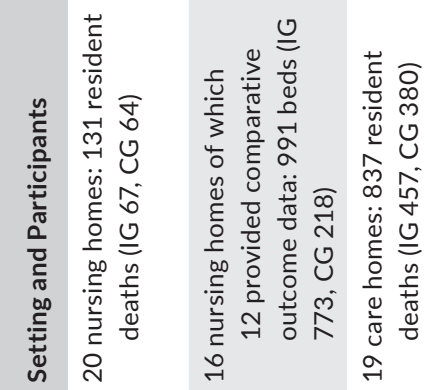

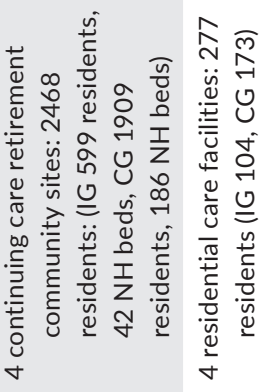

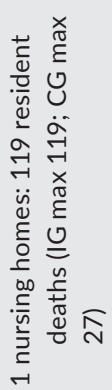

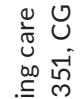
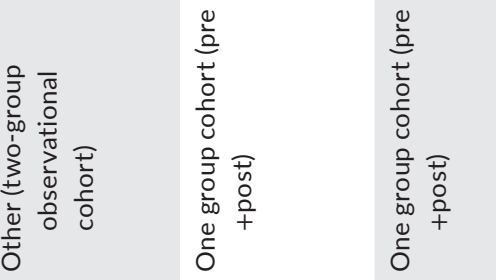

政

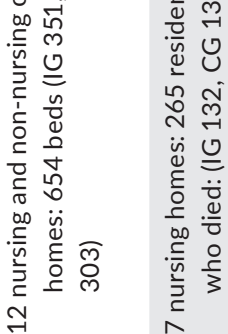
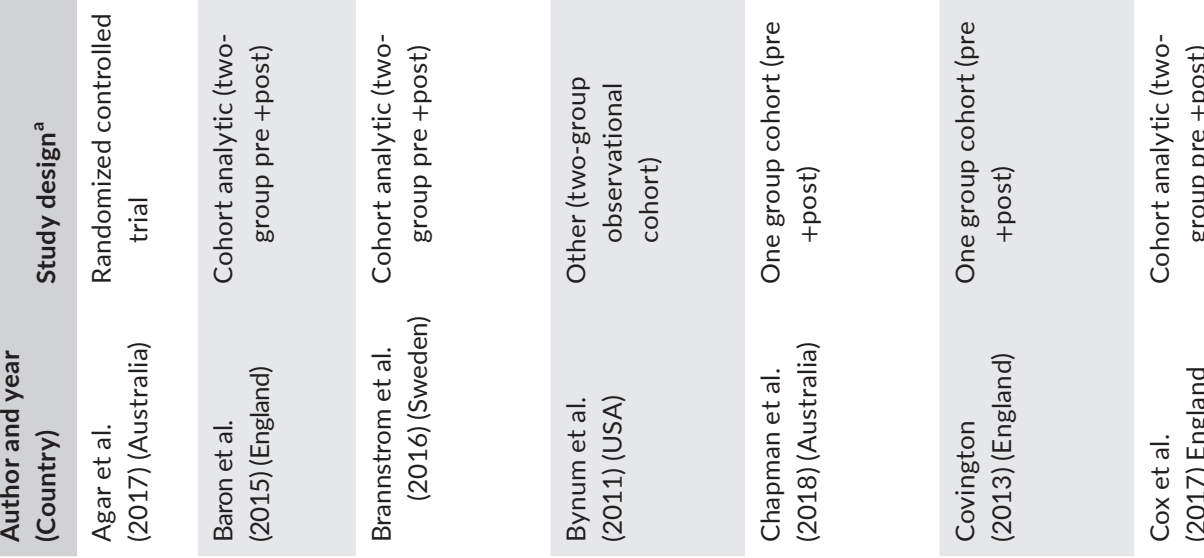

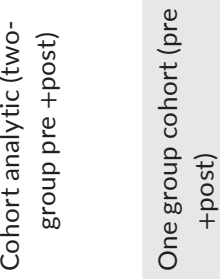

\begin{tabular}{|c|c|c|c|c|c|c|c|c|}
\hline 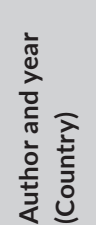 & 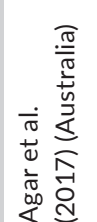 & 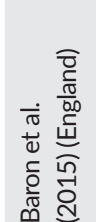 & 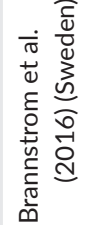 & 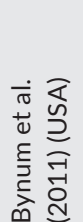 & 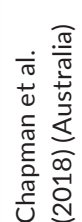 & 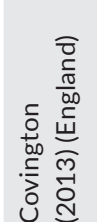 & 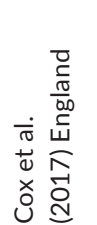 & 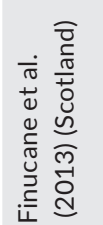 \\
\hline
\end{tabular}




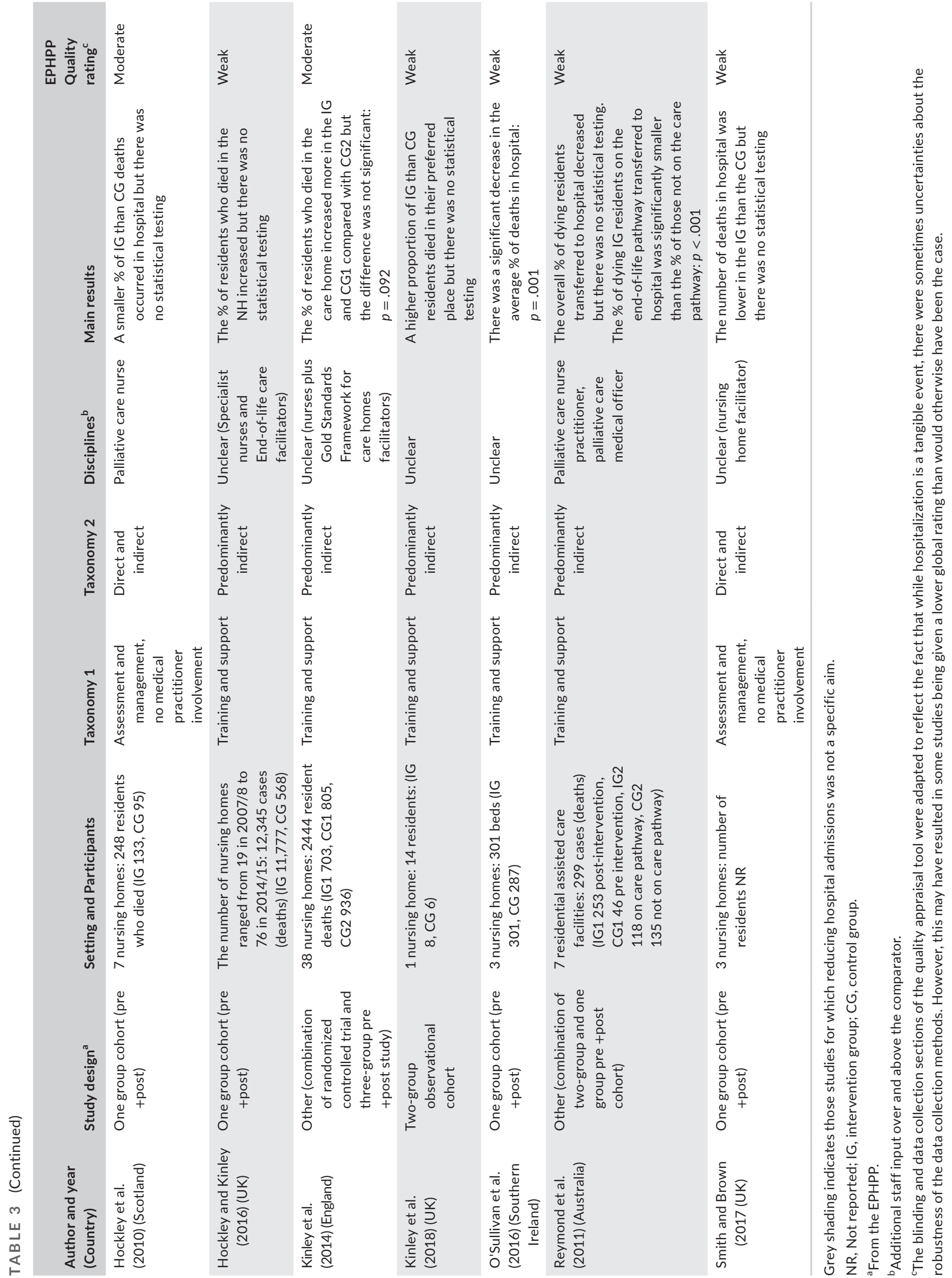


studies in which reported outcomes commonly constituted professional judgements of avoided admissions. Participating facilities included nursing homes, residential homes, assisted living facilities and senior living communities (hereafter 'care homes'). Sample sizes (alternatively reported as numbers of care homes, beds, residents and consultations) varied greatly; the number of care homes ranged from 1 to 405 and the number of residents from 14 to 61,636 , although not all studies provided this information. Most included all older residents. However, some focused on particular subgroups, for example, residents with dementia.

\section{3 | Study quality}

A total of 12 studies were rated strong in quality, 32 moderate and 48 weak. Of the latter, half had weak ratings on two EPHPP domains, 13 on three, 7 on four, and 4 on five. Following EPHPP guidance, 18 weak-rated studies had weak study designs. The three most common reasons for weak classifications were as follows: sample/selection bias (38/48, e.g. low probability participants represented the wider population); data collection methods (33/48, e.g. doubts about data reliability) and the potential for (uncontrolled for) confounders (24/48). Supplementary Appendix S3 provides further details.

\subsection{Focus of the initiatives}

Thirty-two initiatives focused on residents' general physical health, 15 on acute illness or at risk of hospital admission, 20 end-of-life care and 6 post-hospital discharge. Ten sought to optimize residents' medication; others focused on specific health concerns including falls and pressure ulcers.

\subsection{Characteristics of the initiatives}

Not all studies provided detail of participating practitioners. However, at least 46 initiatives were delivered by multidisciplinary teams (MDTs). Nurses (often advanced/specialist practitioners) participated in at least 61 initiatives and physicians at least 49. Other disciplines included pharmacists, occupational therapists, physical therapists, dieticians and emergency care practitioners. Most professional support was provided face to face. However, seven studies evaluated telemedicine services.

Table 4 shows the distribution of the initiatives according to the taxonomies. Employing Taxonomy 1, 55 initiatives provided assessment and management, 13 assessment only and 24 education or training. Using Taxonomy 2, 40 provided predominantly direct care, 26 predominantly indirect care and 26 both.

\subsection{Characteristics of comparator services}

Outcomes of specialist initiatives were typically compared with 'standard' or 'usual' care. However, in a minority, the comparator constituted an earlier iteration of the intervention. In most studies 'usual care' was simply conceptualized as the absence of the additional support the specialist service provided.

\section{7 | Service effectiveness}

\subsection{1 | Effect on hospital admissions/emergency department transfers}

Seventy-five studies that specifically sought to reduce hospital admissions/ED transfers provided data on hospital resource use, of which 11 were rated strong and 24 moderate in quality (Table 2; Supplementary Appendix S2). Eleven studies were entered into a meta-analysis but given the substantial heterogeneity $\left(I^{2}=100 \%\right.$, Figure 2, Forest Plot $\left.A\right)$, the results were not meaningful. Excluding weak studies and studies only providing ED visit data did not markedly reduce the high level of heterogeneity (Forest Plots B and C).

The following subsections synthesize and describe the findings according to the foci of intervention and model of specialist support using vote counting based on direction of effect. Sensitivity analyses are reported only where they cast doubt on the robustness of the main analysis.

\section{General health}

Twenty-seven studies aiming to improve residents' general health provided data on hospital admissions/ED transfers, of which 24 favoured the intervention: $89 \%, 95 \% \mathrm{Cl} 72 \%$ to $96 \%, p<.0001$ ( $p<.001$ corrected for multiple testing), indicating evidence of an effect. One was rated strong in quality, nine moderate and 14 weak. Most provided assessment and management, but two were assessment-only services (Bellantonio et al., 2008; Hex et al., 2015*) and two training and support initiatives (broad quality improvement programmes, Cool et al., 2018; Ouslander et al., 2011; Rolland et al., 2016).

Some of the strongest evidence came from New Zealand, where ED transfers fell $27 \%$ on the introduction of a multidisciplinary support service (Connolly et al., 2018). A similar initiative for residents with 'big five' diagnoses (cardiac failure, ischaemic heart disease, chronic obstructive pulmonary disease, stroke and pneumonia) also reported positive effects (Connolly et al., 2015, 2016), as did a nursing intervention with more emphasis on training (Boyd et al., 2014).

Three other moderately rated studies reporting beneficial outcomes involved both nurses and doctors. In Australia, specialist practice nurses provided assessment, support, education and training with input from a geriatrician (Street et al., 2015); in Hong Kong, community geriatric team nurses assessed all residents, and geriatricians subsequently assessed and reviewed those with complex needs, polypharmacy or frequent readmissions (Luk et al., 2002); in the USA, nurse practitioners and primary care physicians detected problems early and managed them aggressively (Kane et al., 2003, 2004). However, a Dutch initiative by trained nurse assistants with 
WILEY-JAN

TABLE 4 Classification of services by service models

\begin{tabular}{|c|c|c|c|}
\hline \multicolumn{2}{|l|}{ Taxonomy 1} & \multicolumn{2}{|l|}{ Taxonomy 2} \\
\hline Model & Prevalence (n) & Model & Prevalence (n) \\
\hline Assessment, no medical practitioner involvement & 5 & Predominantly direct & 40 \\
\hline Assessment, medical practitioner involvement & 8 & Direct and indirect & 26 \\
\hline $\begin{array}{l}\text { Assessment and management, no medical practitioner } \\
\text { involvement }\end{array}$ & 19 & Predominantly indirect & 26 \\
\hline $\begin{array}{l}\text { Assessment and management, medical practitioner } \\
\text { involvement }\end{array}$ & 36 & & \\
\hline Training and support & 24 & & \\
\hline
\end{tabular}

Forest plot A: meta-analysis of all 11 studies with relevant data

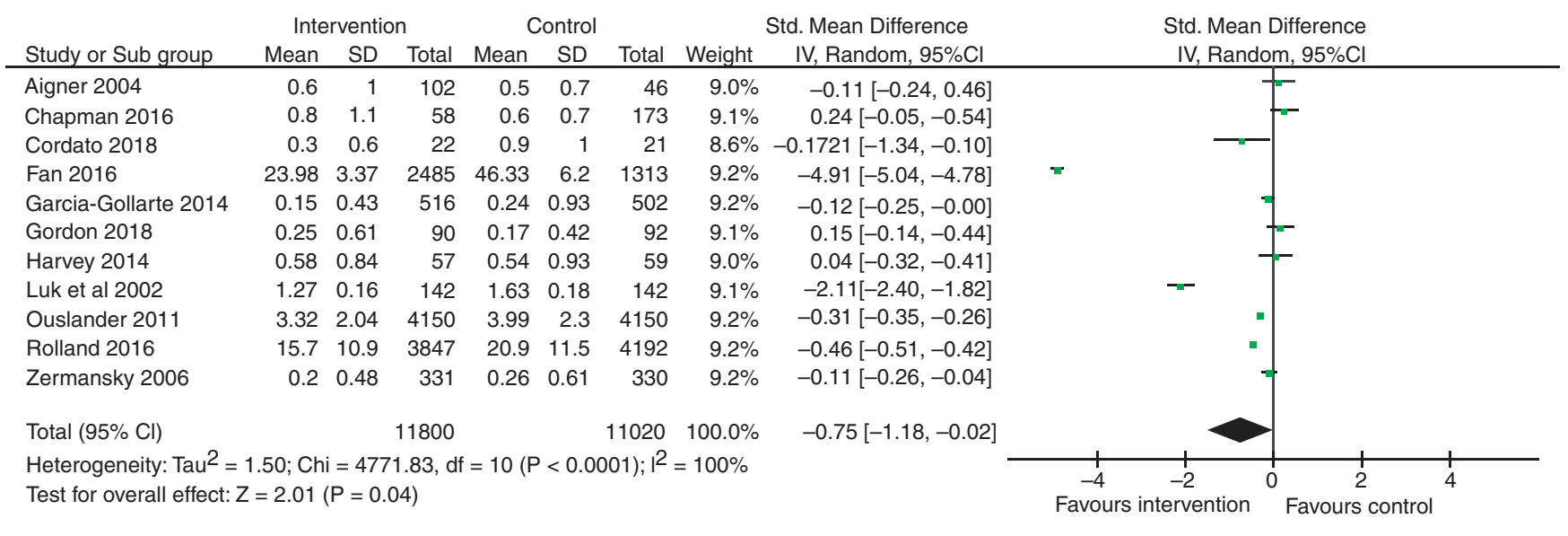

Forest plot B: meta-analysis excluding weak-rated studies

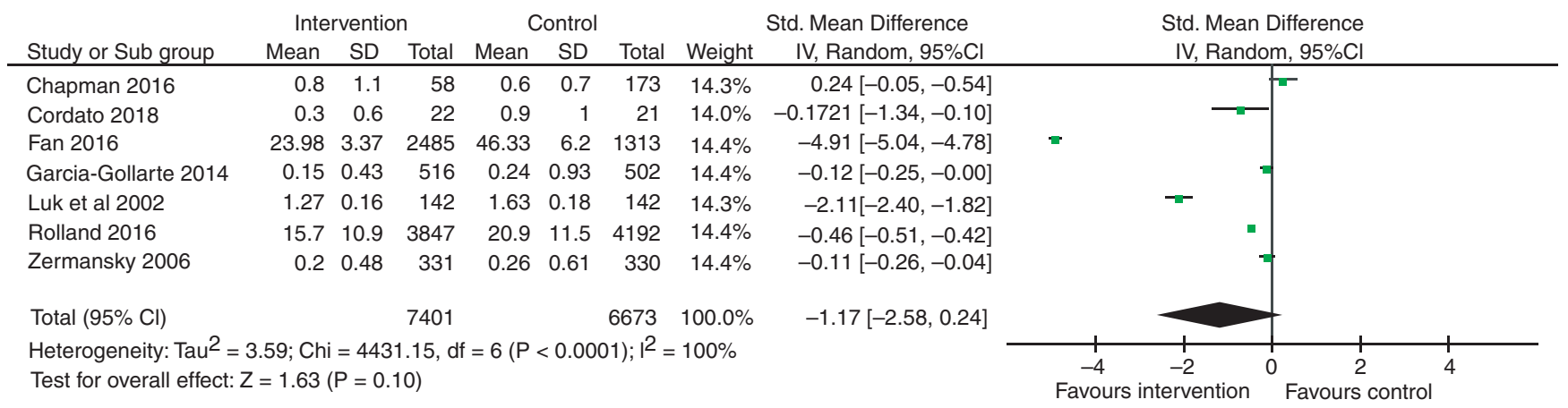

Forest plot C: meta-analysis excluding weak-rated studies and those reporting ED visit data only

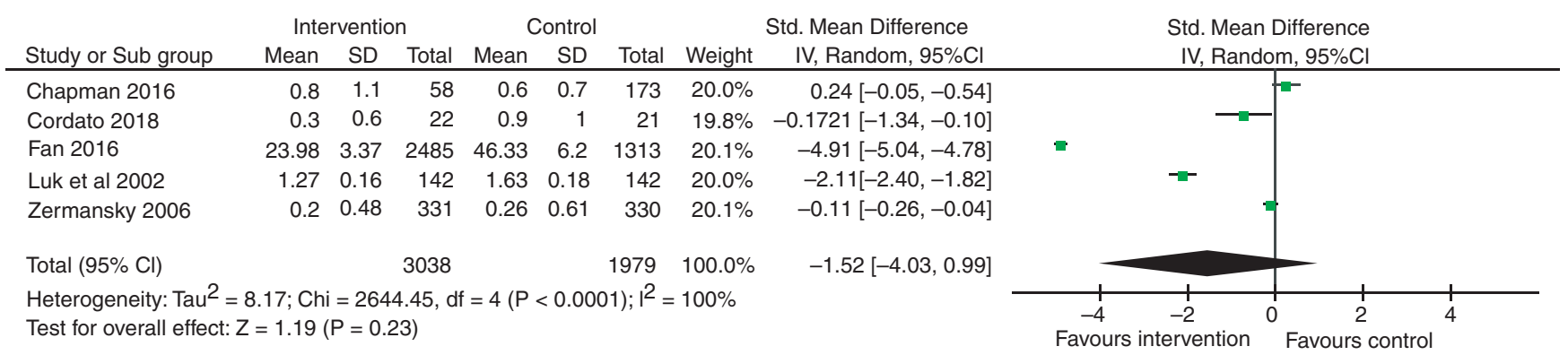

FIGURE 2 Meta-analysis-forest plots of initiatives aiming to reduce hospitalisations 
optional geriatrician/psychologist consultation reported increased admissions (Boorsma et al., 2011).

Four weak-rated multidisciplinary assessment and management initiatives involving doctors and nurses reported reduced admissions (Ackermann \& Kemle, 1998; Hui \& Woo, 2002; Hui et al., 2001; Lisk et al., 2012; Sankaran et al., 2010), as did several other weak-rated enhanced primary care initiatives (Aigner et al., 2004; Burl \& Bonner, 1991; Bynum et al., 2011; Codde et al., 2010; Klaasen et al., 2009; Lacny et al., 2016; Pain et al., 2014). A similar UK intervention, however, reported increased admissions (Gordon et al., 2018*).

Two of three other nurse-staffed services (Arendts et al., 2018; El-Masri et al., 2015; Ono et al., 2015*) and a study of a UK nurse/ pharmacist collaboration (Winstanley \& Brennan, 2007) also reported reduced admissions.

\section{Urgent care}

Thirteen initiatives for acutely unwell residents provided data on ED transfers/hospital admissions, of which 11 reported beneficial outcomes, indicating evidence of effectiveness: $85 \%, 95 \% \mathrm{Cl} 58 \%$ to $0.96 \%, p=.01$. However, this was no longer statistically significant after correction for multiple testing $(p=.06)$. Four were rated strong in quality, two moderate and five weak. Sensitivity analysis treating studies reporting mixed outcomes as negative rather than positive also changed the results so the null hypothesis (no difference between groups) could not be rejected, with 10/13 initiatives favouring the intervention: $77 \%, 95 \% \mathrm{Cl} 50 \%$ to $92 \%, p=.09$.

Two US telemedicine services reported reduced ED transfers/admissions-a nurse-doctor assessment service (Grabowski \& O'Malley, $2014^{*}$ ) and a geriatric medicine provider assessment and management initiative (Shah et al., 2015). A UK assessment-based emergency practitioner service (Mason et al., 2012*) and Canadian paramedic assessment and management service (Jensen et al., 2016*) also reported fewer hospital referrals, albeit both were rated weak.

Of two Australian 'Hospital in the Home' assessment and management of admission-avoidance services, one nursing service failed to demonstrate a clear effect (Crilly et al., 2010). However, the second (strong-rated) medical and nursing initiative reported lower rates of hospital transfer/admissions (Fan et al., 2016), as did several similar services. These included two further strong-rated Australian geriatric outreach services (Chan et al., 2018; Hutchinson et al., 2015), and six moderate-rated US initiatives whereby embedded nurse practitioners provided direct patient care (Ingber et al., 2017; Rantz et al., 2017; Rantz, Popejoy, et al., 2017). A Canadian study in which registered nurses visited care homes on predefined schedules also reported reduced ED transfers (Bandurchin et al., $2011^{*}$ ), but was deemed weak in quality.

Two customized educational initiatives (Hullick et al., 2016; Tena-Nelson et al., 2012*) reported positive effects.

\section{End-of-life care}

Of 11 end-of-life care studies that provided data on hospital transfers/admissions, nine explicitly sought to reduce these (Chapman et al., 2018; Garden et al., 2016; Hockley et al., 2010; Kinley et al., 2018; Kuhn \& Forrest, 2012; Kunte et al., 2017; Luk \& Chan, 2017; O'Sullivan et al., 2016; Teo et al., 2014). Six studies favoured the intervention (Garden et al., 2016; Kinley et al., 2018; Kunte et al., 2017; Luk \& Chan, 2017; O'Sullivan et al., 2016; Teo et al., 2014). However, all were rated weak and the null hypothesis could not be rejected: $66 \%, 95 \% \mathrm{Cl} 0.35$ to $0.88, p=.25$. Section 3.7.2 provides more information about these services' effect on place of death.

\section{Medication}

Of ten studies aiming to improve prescribing, six sought to reduce hospitalization and provided data on ED transfer/admission numbers (García-Gollarte et al., 2014; Lapane, Hughes, Christian, et al., 2011; Lapane et al., 2011; McKee et al., 2016; Roberts et al., 2001; Zermansky et al., 2006). Of these, four favoured the intervention (one strong, two moderate and one weak). Three involved medication reviews by clinical pharmacists (Lapane, Hughes, Christian, et al., 2011; McKee et al., 2016; Zermansky et al., 2006) and the fourth a 10-hour training programme (García-Gollarte et al., 2014). However, synthesis suggested the null hypothesis could not be rejected: $66 \%, 95 \% \mathrm{Cl} 30 \%$ to $90 \%, p=.34$.

\section{Post-hospital discharge}

All six studies evaluating post-hospital discharge services reported decreased admissions/re-admissions, suggesting a positive effect: $100 \%, 95 \% \mathrm{Cl} 61 \%$ to $100 \%, p=.015$. However, this was no longer statistically significant after correction for multiple testing $(p=.09)$. One was rated strong, three moderate and two weak. When studies reporting mixed findings were classed as negative rather than positive, the null hypothesis could also no longer be rejected, although 5/6 studies favoured the intervention: $83 \%, 95 \% \mathrm{Cl} 44 \%$ to $97 \%$, $p=.11$.

Of three Danish studies, one evaluated a rehabilitation intervention post hip fracture provided by a geriatric orthopaedic team (Gregersen et al., 2011) and two investigated general health support services provided by nurse-physician geriatric teams (Pedersen et al., 2016, 2018). Each assessed care home residents on the first working day post-discharge, while the services available in the general health initiatives variously included telephone advice, blood tests, electrocardiograms, intravenous antibiotics, oxygen, subcutaneous fluids and blood transfusions (Pedersen et al., 2016, 2018). Two further initiatives also provided assessment and management (Harvey et al., 2014; Schubert et al., 2016), while in the sixth, a geriatrician/nurse practitioner assessment service, the initial visit was somewhat later than in the Danish studies, but support continued longer (Cordato et al., 2018).

\section{Other specialist initiatives}

Five training initiatives focused on preventing falls/hip fracture (Walker et al., 2016; Ward et al., 2010); falls, pressure ulcers and incontinence (Brownhill, 2013); pneumonia (Hutt et al., 2011) and the provision of intravenous fluids/antibiotics (Romøren et al., 2017) with a view to reducing hospitalization. Three favoured the 
intervention (Brownhill, 2013; Hutt et al., 2011; Romøren et al., 2017). However, synthesis suggested that the null hypothesis could not be rejected: $60 \%, 95 \% \mathrm{Cl} 23 \%$ to $88 \%, p=.5$.

\section{Taxonomies of specialist care home support}

Taxonomy 1. Seven of nine assessment-only interventions (with/ without medical practitioner involvement) (78\%, $95 \% \mathrm{Cl} 45 \%$ to $94 \%$, $p=.09)$ favoured the intervention. Thus, the null hypotheses could not be rejected. There was, however, evidence that assessment and management interventions (with/without medical practitioner involvement) had an effect, with 36 of 43 studies favouring the intervention: $84 \%, 95 \% \mathrm{Cl} 70 \%$ to $92 \%, p<.00001$ ( $p<.0001$ when corrected for multiple testing). Syntheses of all non-training initiatives with medical practitioner involvement also showed evidence of effectiveness, with $32 / 36$ studies favouring the intervention: $89 \%$, $95 \% \mathrm{Cl} 75 \%$ to $96 \%, p<.00001$ ( $p<.0001$ when corrected for multiple testing). Conversely, synthesis of non-training interventions without medical involvement failed to reject the null hypothesis, 11 of 16 studies favouring the intervention: $69 \%, 95 \% \mathrm{Cl} 44 \%$ to $86 \%$, $p=.10$. Synthesis of training only initiatives (with/without medical practitioner involvement) also showed evidence of effectiveness, with 11 of 14 favouring the intervention: $78 \%, 95 \% \mathrm{Cl} 52 \%$ to $92 \%$, $p=.029$. However, this was no longer statistically significant after correction for multiple testing ( $p=.15$ ).

Taxonomy 2. Twenty-eight of 30 interventions providing predominantly direct care favoured the intervention, showing evidence of an effect: $93 \%, 95 \% \mathrm{Cl} 79 \%$ to $98 \%, p<.00001$ ( $p=.00003$ when corrected for multiple testing). Predominantly indirect care also showed an effect in the main analyses, with 12/15 studies favouring the intervention: $80 \%, 95 \% \mathrm{Cl} 55 \%$ to $93 \%, p=.017$ ( $p=.05$ when corrected for multiple testing), but this was not sustained in sensitivity analyses: $67 \%, 95 \% \mathrm{Cl} 42 \%$ to $85 \%, p=.15$. Synthesis of interventions providing direct and indirect care failed to reject the null hypothesis, 14 of 21 studies favouring the intervention: $67 \%$, $95 \% \mathrm{Cl} 45 \%$ to $83 \%, p=.09$.

\subsection{2 | Effect on place of death}

Fifteen studies provided data on residents' place of death, of which 14 aimed to reduce hospital admissions (Table 3; Supplementary Appendix S3). All evaluated end-of-life care initiatives, with five considered moderate and nine weak in quality. Nine provided training and support in palliative care and/or advance care planning (Baron et al., 2015; Brännström et al., 2016; Covington, 2013; Cox et al., 2017; Hockley \& Kinley, 2016; Kinley et al., 2014, 2018; O'Sullivan et al., 2016; Reymond et al., 2011), of which four drew on the Gold Standards Framework quality improvement training programmes (Baron et al., 2015; Covington, 2013; Hockley \& Kinley, 2016; Kinley et al., 2014), and others the (subsequently phased out) Liverpool Care Pathway (Brännström et al., 2016; Kinley et al., 2014; Reymond et al., 2011). The remainder involved training and assessment and/ or management by external care practitioners (generally nurses)
(Bynum et al., 2011; Chapman et al., 2018; Finucane et al., 2013; Hockley et al., 2010; Smith \& Brown, 2017). Overall, 13/14 studies favoured the intervention, demonstrating evidence of effectiveness: $93 \%, 95 \% \mathrm{Cl} 68 \%$ to $99 \%, p<.001$.

\section{8 $\quad$ Healthcare expenditure}

Thirty-four studies provided service cost information (Supplementary Appendix S4). However, only two undertook full cost-effectiveness analyses (Lacny et al., 2016; Sackley et al., 2016). Neither had a significant effect on hospital admissions and only one explicitly aimed to reduce admissions (Lacny et al., 2016).

Of 19 moderate-/strong-rated studies with significant effects on transfers/admissions, five provided cost data. Each reported savings (Boyd et al., 2014; Chan et al., 2018; Cordato et al., 2018; Ingber et al., 2017; Kane et al., 2003, 2004), but only three undertook formal cost evaluations. The total costs (inpatient, ED presentations and physician visit costs) of an Australian post-hospital discharge service (Cordato et al., 2018) were \$91,854 (mean \$4175) compared with $\$ 175,517$ (mean $\$ 8358$ ) for the control group, while an Australian Acute Geriatric Outreach Service reported average savings of $\$ 2353$ per person from reduced admission and ambulance transfer frequency (Chan et al., 2018). In seven US urgent care initiatives, average per resident Medicare expenditures reduced by $\$ 60-$ $\$ 2248$ for all-cause hospitalizations and $\$ 98-\$ 577$ for potentially avoidable hospitalizations (Ingber et al., 2017).

\section{4 | DISCUSSION}

This comprehensive, systematic review identified 92 studies of specialist healthcare support for care homes and highlighted their potential effectiveness in preventing the hospitalization of care home residents. Reflecting growing concern for this population, most studies were published in the last decade, and compared with earlier reviews (Davies et al., 2011; Goldman, 2013; Graverholt et al., 2014; Santosaputri et al., 2018), the proportion of moderate-/strong-quality studies was high, suggesting the emergence of better-quality evidence. Evidence (albeit not definitive) was found for interventions addressing residents' general health needs, assessment and management services and non-training initiatives involving medical staff. Promising evidence was also found for services targeting residents at imminent risk of hospital entry or posthospital discharge and training-only initiatives. However, sensitivity analysis (correcting for multiple testing) suggested these results should be treated with caution. Similarly, although evidence was found for the ability of end-of-life services to enable residents to remain in the care home at end of life, the quality of the individual studies that explored this was generally weak, undermining confidence in the results.

The results of this review are important in informing future service design. The use of the taxonomies representing different 
organizational models has specific implications for clinical practice. Considering Taxonomy 1, stronger evidence was found for services undertaking assessment and management than assessment alone, demonstrating the importance of specialist healthcare services diagnosing and treating the healthcare needs of care home residents in partnership with care home staff. This message was reinforced by evidence for services providing predominantly direct care using Taxonomy 2, and by implication means specialist healthcare staff must be available to care home residents on a regular, if not daily basis. The analysis also showed evidence for the effectiveness of training-only initiatives, with potentially significant impact for service costs. Specialist services might, for example, deliver training for staff providing hands on care for acutely unwell residents rather than providing direct care themselves. Interestingly, no clear evidence was found for the effectiveness of services providing direct and indirect care, suggesting services may find it difficult to do both well simultaneously.

Another important finding is the association between the involvement of medical practitioners/MDTs and more positive effects, reinforcing the findings of previous reviews (Graverholt et al., 2014; Santosaputri et al., 2018), with obvious implications for service staffing and multidisciplinary working. In highlighting the potential effectiveness of interventions targeted at residents on the margins of hospital care (at risk of hospital admission or post-discharge), the review also highlights the skills and knowledge required to limit avoidable admissions/readmissions, including expertise in proactively identifying, monitoring and managing acutely unwell residents, administering intravenous fluids and drugs.

The importance of enabling residents to remain in their care home at the end of life is acknowledged (Social Care Institute for Excellence, 2017). The suggestion that specialist care home support services can have a positive effect on this outcome is, thus, encouraging. However, although the review identified 15 initiatives reporting place-of-death outcomes, the quality of evidence was generally weak and, individually, only one moderate-rated study reported a significant effect (Chapman et al., 2018).

Overall, most studies were complex interventions characterized by several interacting components tailored to local circumstances rather than being standardized. Over a quarter were randomized/ controlled trials, showing such designs are feasible. However, if using other designs, steps to enhance quality include accounting for potential confounders and conducting appropriate statistical tests. Definitions of usual/standard care and data on service costs would also enable stakeholders to make better informed decisions. As the evidence base for the provision of specialist healthcare support services for care homes grows, researchers should employ the fourphase framework for complex interventions (development, feasibility/piloting, evaluation and implementation) to guide intervention development (Craig et al., 2008; O'Cathain et al., 2019).

In reviewing the support required by older care home residents, one cannot ignore the ongoing Covid-19 pandemic (Burton et al., 2020; Davidson \& Szanton, 2020); 'usual care' has not been usual for anyone of late, particularly care home staff/residents. This review was conducted before the pandemic emerged. However, older people are particularly vulnerable to the virus and this has rapidly impacted both clinical practice and research (Richardson et al., 2020). While opportunities for new specialist support initiatives may be curtailed, the need to avoid hospitalization is accentuated. Tailored training for care home staff and access to specialist consultation via telephone/online may become more valuable, and necessary, than ever.

\section{1 | Methodological considerations}

Several methodological considerations must be taken into account when assessing these findings. First, although searches were undertaken in a broad range of databases and the review encompassed a wide spectrum of initiatives, the quality of the evidence was mixed. Many studies involved small numbers of care homes or failed to report sample sizes; most provided little information about comparator services and some lacked detail of the initiative itself. Second, although the taxonomies offered an opportunity to explore the effectiveness of different service configurations, there was inevitably still some subjectivity in the categorization of services and heterogeneity in categorizations. Third, by restricting inclusions to peer-reviewed studies in the English language, the review potentially overlooked other relevant research and the findings may be less generalizable to care home residents in parts of the world with different service structures. Fourth, the possibility of publication bias was not formally assessed and the potential for this cannot be ruled out. Fifth, given the high level of heterogeneity among studies, meta-analysis results were not meaningful. Vote counting by direction of effect facilitated inclusion of the maximum number of studies. However, while this can answer the question 'Is there evidence of an effect?', it provides no information on the magnitude of effects and does not account for differences in study size (McKenzie \& Brennan, 2019), such that the results should be treated with caution.

Going forward, it is suggested that researchers use the Template for Intervention Description and Replication (TIDiER) checklist when reporting individual studies to improve the completeness and quality of reporting (Hoffman et al., 2014), while future reviews would also benefit from employment of the Grading of Recommendations Assessment, Development and Evaluation (GRADE) approach (Schünemann et al., 2021). This increasingly widely used tool summarizes confidence in the effects of interventions across studies based on consideration of five domains (risk of bias, inconsistency, indirectness, imprecision and publication bias) and specifies four levels of certainty for any given outcome - high, moderate, low and very low.

\section{5 | CONCLUSION}

This review has synthesized the evidence on the ability of specialist healthcare support services for care homes to prevent avoidable 
hospital admissions, although few studies provided cost information, an area for future research. The classification of studies by both foci of intervention and organizational model permitted similar interventions to be appraised together, providing a marker of the strength of the evidence for different service subgroups. A focus on the three components of service delivery identified in this review - multidisciplinary collaboration, including medical practitioners; expertise in diagnosis, monitoring and managing residents (including acutely unwell residents) and training for care home staff - and how they might be combined to best effect is relevant to care home providers, service planners, researchers and care home residents.

\section{ACKNOWLEDGEMENTS}

We thank Jennifer Boland for her help with the initial screening process and Vincent Gillan for his administrative support of the study.

\section{CONFLICTS OF INTEREST}

No conflict of interest has been declared by the authors.

\section{AUTHOR CONTRIBUTIONS}

All authors have agreed on the final version and meet at least one of the following criteria (recommended by the ICMJE [http://www. icmje.org/recommendations/]): substantial contributions to conception and design, acquisition of data or analysis and interpretation of data and drafting the article or revising it critically for important intellectual content.

\section{PEER REVIEW}

The peer review history for this article is available at https://publo ns.com/publon/10.1111/jan.15043.

\section{DATA AVAILABILITY STATEMENT}

The data that support the findings of this study are openly available in public repository 'Figshare' at https://doi.org/10.48420/16548 216.v1.

\section{ORCID}

Deborah Buck (D) https://orcid.org/0000-0002-2271-6400

Sue Tucker (D) https://orcid.org/0000-0001-7986-0844

Brenda Roe (D) https://orcid.org/0000-0001-8227-0116

Jane Hughes (D) https://orcid.org/0000-0002-6158-1211

David Challis (D) https://orcid.org/0000-0002-6464-2286

\section{REFERENCES}

Ackermann, R. J., \& Kemle, K. A. (1998). The effect of a physician assistant on the hospitalization of nursing home residents. Journal of the American Geriatrics Society, 46, 610-614. https://doi.org/10.1111/ j.1532-5415.1998.tb01078.x

Agar, M., Luckett, T., Luscombe, G., Phillips, J., Beattie, E., Pond, D., Mitchell, G., Davidson, P. M., Cook, J., Brooks, D., Houltram, J., Goodall, S., \& Chenoweth, L. (2017). Effects of facilitated family case conferencing for advanced dementia: A cluster randomised clinical trial. PLoS One, 12, e0181020. https://doi.org/10.1371/ journal.pone.0181020
Agotnes, G., Jacobsen, F. F., Harrington, C., \& Petersen, K. A. (2016). A critical review of research on hospitalization from nursing homes; What is missing? Ageing International, 41, 3-16. https://doi. org/10.1007/s12126-015-9232-4

Aigner, M. J., Drew, S., \& Phipps, J. (2004). A comparative study of nursing home resident outcomes between care provided by nurse practitioners/physicians versus physicians only. Journal of the American Medical Directors Association, 5, 16-23. https://doi.org/10.1016/ S1525-8610(04)70039-0

Alldred, D. P., Kennedy, M. C., Hughes, C., Chen, T. F., \& Miller, P. (2016). Interventions to optimise prescribing for older people in care homes. The Cochrane Database of Systematic Reviews, 2, CD009095. https://doi.org/10.1002/14651858.CD009095.pub3

Arendts, G., Deans, P., O'Brien, K., Etherton-Beer, C., Howard, K., Lewin, G., \& Sim, M. (2018). A clinical trial of nurse practitioner care in residential aged care facilities. Archives of Gerontology and Geriatrics, 77, 129-132. https://doi.org/10.1016/j.archger.2018.05.001

Arendts, G., Jan, S., Beck, M. J., \& Howard, K. (2017). Preferences for the emergency department or alternatives for older people in aged care: A discrete choice experiment. Age and Ageing, 46, 124-129. https://doi.org/10.1093/ageing/afw163

Armijo-Olivo, S., Stiles, C. R., Hagen, N. A., Biondo, P. D., \& Cummings, G. C. (2012). Assessment of study quality for systematic reviews: A comparison of the Cochrane Collaboration risk of bias tool and the effective public health practice project quality assessment tool: Methodological research. Journal of Evaluation in Clinical Practice, 18, 12-18. https://doi.org/10.1111/j.1365-2753.2010.01516.x

Bandurchin, A., McNally, M. J., \& Ferguson-Paré, M. (2011). Bringing back the house call: How an emergency mobile nursing service is reducing avoidable emergency department visits for residents in long-term care homes. Nursing Leadership, 24, 59-71. https://doi. org/10.12927/cjnl.2011.22335

Baron, K., Hodgson, A., \& Walshe, C. (2015). Evaluation of an advance care planning education programme for nursing homes: A longitudinal study. Nurse Education Today, 35, 689-695. https://doi. org/10.1016/j.nedt.2015.01.005

Beck, A. M., Christensen, A. G., Hansen, B. S., Damsbo-Svendsen, S., \& Møller, T. K. (2016). Multidisciplinary nutritional support for undernutrition in nursing home and home-care: A cluster randomized controlled trial. Nutrition, 32, 199-205. https://doi.org/10.1016/j. nut.2015.08.009

Bellantonio, S., Kenny, A. M., Fortinsky, R. H., Kleppinger, A., Robison, J., Gruman, C., Kulldorff, M., \& Trella, P. M. (2008). Efficacy of a geriatrics team intervention for residents in dementia-specific assisted living facilities: Effect on unanticipated transitions. Journal of the American Geriatrics Society, 56, 523-528. https://doi. org/10.1111/j.1532-5415.2007.01591.x

Boorsma, M., Frijters, D. H., Knol, D. L., Ribbe, M. E., Nijpels, G., \& van Hout, H. P. (2011). Effects of multidisciplinary integrated care on quality of care in residential care facilities for elderly people: A cluster randomized trial. Canadian Medical Association Journal, 183, E724-E732. https://doi.org/10.1503/cmaj.101498.

Borenstein, M., Hedges, L. V., Higgins, J. P., \& Rothstein, H. R. (2010). A basic introduction to fixed-effect and random-effects models for meta-analysis. Research Synthesis Methods, 1(2), 97-111. https:// doi.org/10.1002/jrsm.12

Boyd, M., Armstrong, D., Parker, J., Pilcher, C., Zhou, L., McKenzie-Green, B., \& Connolly, M. J. (2014). Do gerontology nurse specialists make a difference in hospitalization of long-term care residents? Results of a randomized comparison trial. Journal of the American Geriatrics Society, 62, 1962-1967. https://doi.org/10.1111/jgs.13022

Brännström, M., Fürst, C. J., Tishelman, C., Petzold, M., \& Lindqvist, O. (2016). Effectiveness of the Liverpool care pathway for the dying in residential care homes: An exploratory, controlled before-and-after study. Palliative Medicine, 30, 54-63. https://doi. org $/ 10.1177 / 0269216315588007$ 
British Geriatrics Society. (2016). Effective healthcare for older people living in care homes. Guidance on commissioning and providing healthcare services across the UK. Retrieved October 24, 2020, from https://www.bgs.org.uk/sites/default/files/content/resou rces/files/2018-05-10/2016_bgs_commissioning_guidance.pdf

Brownhill, K. (2013). Training in care homes to reduce avoidable harm. Nursing Times, 109, 20-22.

Burl, J. B., \& Bonner, A. F. (1991). A geriatric nurse practitioner/physician team in a long-term care setting. HMO Practice, 5, 139-142.

Burl, J. B., Bonner, A., Rao, M., \& Khan, A. M. (1998). Geriatric nurse practitioners in long-term care: Demonstration of effectiveness in managed care. Journal of the American Geriatrics Society, 46, 506-510. https://doi.org/10.1111/j.1532-5415.1998.tb02475.x

Burton, J. K., Bayne, G., Evans, C., Garbe, F., Gorman, D., Honhold, N., McCormick, D., Othieno, R., Stevenson, J., Swietlik, S., Templeton, K. E., Tranter, M., Willocks, L., \& Guthrie, B. (2020). Evolution and effects of COVID-19 outbreaks in care homes: A population analysis in 189 care homes in one geographical region of the UK. Lancet Healthy Longevity, 1, e21-e31. https://doi.org/10.1016/S2666 -7568(20)30012-X

Bynum, J. P., Andrews, A., Sharp, S., McCollough, D., \& Wennberg, J. E. (2011). Fewer hospitalizations result when primary care is highly integrated into a continuing care retirement community. Health Affairs, 30, 975-984. https://doi.org/10.1377/hlthaff.2010.1102

Campbell, M., McKenzie, J. E., Sowden, A., Katikireddi, S. V., Brennan, S. E., Ellis, S., Hartmann-Boyce, J., Ryan, R., Shepperd, S., Thomas, J., Welch, V., \& Thomson, H. (2020). Synthesis without meta-analysis (SWiM) in systematic reviews: Reporting guideline. British Medical Journal, 368, 16890. https://doi.org/10.1136/bmj.16890

(2009). Systematic reviews. CRD's guidance for undertaking reviews in health care. Centre for Reviews and Dissemination. Retrieved October 20, 2020, from http://www.york.ac.uk/crd/guidance/

Chan, D. K. Y., Liu, F. X., Irwanto, D., Prasetyo, D., Ozorio, G., Li, F., Smith, M., Sharma, A., \& Chen, J. (2018). Experience of establishing an acute geriatric outreach service versus subacute service to nursing homes. Internal Medicine Journal, 48, 1396-1399. https://doi. org/10.1111/imj.14104

Chapman, M., Johnston, N., Lovell, C., Forbat, L., \& Liu, W. M. (2018). Avoiding costly hospitalisation at end of life: Findings from a specialist palliative care pilot in residential care for older adults. BMJ Supportive \& Palliative Care, 8, 102-109. https://doi.org/10.1136/ bmjspcare-2015-001071

Clarkson, P., Hays, R., Tucker, S., Paddock, K., \& Challis, D. (2018). Healthcare support to older residents of care homes: A systematic review of specialist services. Quality in Ageing and Older Adults, 19, 54-84. https://doi.org/10.1108/QAOA-08-2017-0029

Codde, J., Arendts, G., Frankel, J., Ivey, M., Reibel, T., Bowen, S., \& Babich, P. (2010). Transfers from residential aged care facilities to the emergency department are reduced through improved primary care services: an intervention study. Australasian Journal on Ageing, 29, 150154. https://doi.org/10.1111/j.1741-6612.2010.00418.x

Connolly, M. J., Boyd, M., Broad, J., Kerse, N., Lumley, T., Whitehead, N., \& Foster, S. (2015). The Aged Residential Care Healthcare Utilization Study (ARCHUS): A multidisciplinary, cluster randomized controlled trial designed to reduce acute avoidable hospitalizations from long-term care facilities. Journal of the American Medical Directors Association, 16, 49-55. https://doi.org/10.1016/j. jamda.2014.07.008

Connolly, M. J., Broad, J. B., Bish, T., Zhang, X., Bramley, D., Kerse, N., Bloomfield, K., \& Boyd, M. (2018). Reducing emergency presentations from long-term care: A before-and-after study of a multidisciplinary team intervention. Maturitas, 117, 45-50. https://doi. org/10.1016/j.maturitas.2018.08.014

Connolly, M. J., Broad, J. B., Boyd, M., Zhang, T. X., Kerse, N., Foster, S., Lumley, T., \& Whitehead, N. (2016). The 'Big Five'. Hypothesis generation: a multidisciplinary intervention package reduces disease-specific hospitalisations from long-term care: A post hoc analysis of the ARCHUS cluster-randomised controlled trial. Age and Ageing, 45, 415-420. https://doi.org/10.1093/ageing/afw037

Cool, C., Cestac, P., McCambridge, C., Rouch, L., de Souto Barreto, P., Rolland, Y., \& Lapeyre-Mestre, M. (2018). Reducing potentially inappropriate drug prescribing in nursing home residents: Effectiveness of a geriatric intervention. British Journal of Clinical Pharmacology, 84, 1598-1610. https://doi.org/10.1111/bcp.13598

Cordato, N. J., Kearns, M., Smerdely, P., Seeher, K. M., Gardiner, M. D., \& Brodaty, H. (2018). Management of nursing home residents following acute hospitalization: Efficacy of the "Regular Early Assessment Post-Discharge (REAP)" intervention. Journal of the American Medical Association, 19, 276.e11-276.e19. https://doi. org/10.1016/j.jamda.2017.12.008

Covington, M. (2013). End-of-life care: Implementing the Gold Standards Framework. Nursing and Residential Care, 15, 146-149. https://doi. org/10.12968/nrec.2013.15.3.146

Cox, A., Arber, A., Bailey, F., Dargan, S., Gannon, C., Lisk, R., Quinn, B., Samarasinghe, J., Wrigley, M., \& Gallagher, A. (2017). Developing, implementing and evaluating an end of life care intervention. Nursing Older People, 29, 27-35. https://doi.org/10.7748/nop.2017. e873

Craig, P., Dieppe, P., Macintyre, S., Michie, S., Nazareth, I., \& Petticrew, M. (2008). Developing and evaluating complex interventions: The new Medical Research Council guidance. British Medical Journal, 337, a1655. https://doi.org/10.1136/bmj.a1655

Crilly, J., Chaboyer, W., Wallis, M., Thalib, L., \& Polit, D. (2010). An outcomes evaluation of an Australian Hospital in the Nursing Home admission avoidance programme. Journal of Clinical Nursing, 20, 1178-1187. https://doi.org/10.1111/j.1365-2702.2010.03371.x

Davidson, P. M., \& Szanton, S. L. (2020). Nursing homes and COVID-19: We can and should do better. Journal of Clinical Nursing, 29, 27582759. https://doi.org/10.1111/jocn.15297

Davies, S. L., Goodman, C., Bunn, F., Victor, C., Dickinson, A., Iliffe, S., Gage, H., Martin, W., \& Froggatt, K. (2011). A systematic review of integrated working between care homes and health care services. BMC Health Services Research, 11, 320. https://doi. org/10.1186/1472-6963-11-320

Deloitte Access Economics. (2020). Aged Care Reform: Projecting Future Impacts. Research paper 11. Retrieved August 7, 2021, from https://agedcare.royalcommission.gov.au/sites/default/files/ 2020-09/research-paper-11-aged-care-reform-projecting-futureimpacts.pdf

El-Masri, M. M., Omar, A., \& Groh, E. M. (2015). Evaluating the effectiveness of a nurse practitioner-led outreach program for longterm-care homes. Canadian Journal of Nursing Research, 47, 39-55. https://doi.org/10.1177/084456211504700304

Ennis, L., Kinley, J., Hockley, J., \& McCrone, P. (2015). The cost of providing end of life care for nursing care home residents: A retrospective cohort study. Health Services Management Research, 28, 16-23. https://doi.org/10.1177/0951484815607541

Fan, L., Hou, X. Y., Zhao, J., Sun, J., Dingle, K., Purtill, R., Tapp, S., \& Lukin, B. (2016). Hospital in the Nursing Home program reduces emergency department presentations and hospital admissions from residential aged care facilities in Queensland, Australia: A quasiexperimental study. BMC Health Services Research, 16, 46. https:// doi.org/10.1186/s12913-016-1275-z

Feldstein, D., Sloane, P. D., \& Feltner, C. (2018). Antibiotic Stewardship Programs in nursing homes: A systematic review. Journal of the American Medical Association, 19, 110-116. https://doi. org/10.1016/j.jamda.2017.06.019

Finucane, A. M., Stevenson, B., Moyes, R., Oxenham, D., \& Murray, S. A. (2013). Improving end-of-life care in nursing homes: Implementation and evaluation of an intervention to sustain quality of care. Palliative Medicine, 27, 772-778. https://doi.org/10.1177/02692 16313480549 
Forsetlund, L., Eike, M. C., Gjerberg, E., \& Vist, G. E. (2011). Effect of interventions to reduce potentially inappropriate use of drugs in nursing homes: A systematic review of randomised controlled trials. BMC Geriatrics, 11, 16. https://doi.org/10.1186/1471-2318-11-16

Furniss, L., Burns, A., Craig, S. K., Scobie, S., Cooke, J., \& Faragher, B. (2000). Effects of a pharmacist's medication review in nursing homes. Randomised controlled trial. British Journal of Psychiatry, 176, 563-567. https://doi.org/10.1192/bjp.176.6.563

García-Gollarte, F., Baleriola-Júlvez, J., Ferrero-López, I., Cuenllas-Díaz, Á., \& Cruz-Jentoft, A. J. (2014). An educational intervention on drug use in nursing homes improves health outcomes resource utilization and reduces inappropriate drug prescription. Journal of the American Medical Directors Association, 15, 885-891. https://doi org/10.1016/j.jamda.2014.04.010

Garden, G., Green, S., Pieniak, S., \& Gladman, J. (2016). The Bromhead Care Home Service: The impact of a service for care home residents with dementia on hospital admission and dying in preferred place of care. Clinical Medicine, 16, 114-118. https://doi.org/10.7861/clinm edicine.16-2-114

Goldman, R. (2013). Evidence review on partnership working between GPs, care home residents and care homes. Social Care Institute for Excellence.

Gordon, A. L., Franklin, M., Bradshaw, L., Logan, P., Elliott, R., \& Gladman, J. R. (2014). Health status of UK care home residents: A cohort study. Age and Ageing, 43, 97-103. https://doi.org/10.1093/agein g/aft077

Gordon, A. L., Goodman, C., Davies, S. L., Dening, T., Gage, H., Meyer, J., Schneider, J., Bell, B., Jordan, J., Martin, F. C., Iliffe, S., Bowman, C., Gladman, J. R. F., Victor, C., Mayrhofer, A., Handley, M., \& Zubair, M. (2018). Optimal healthcare delivery to care homes in the UK: A realist evaluation of what supports effective working to improve healthcare outcomes. Age and Ageing, 47, 595-603. https://doi. org/10.1093/ageing/afx195

Grabowski, D. C., \& O'Malley, A. J. (2014). The care span: Use of telemedicine can reduce hospitalizations of nursing home residents and generate savings for medicare. Health Affairs, 33, 244-250. https:// doi.org/10.1377/hlthaff.2013.0922

Graverholt, B., Forsetlund, L., \& Jamtvedt, G. (2014). Reducing hospital admissions from nursing homes: A systematic review. BMC Health Services Research, 14, 36. https://doi. org/10.1186/1472-6963-14-36.

Gregersen, M., Zintchouk, D., Borris, L. C., \& Damsgaard, E. M. (2011). A geriatric multidisciplinary and tailor-made hospital-at-home method in nursing home residents with hip fracture. Geriatric Orthopaedic Surgery and Rehabilitation, 2, 148-154. https://doi. org/10.1177/2151458511421908

Hall, S., Kolliakou, A., Petkova, H., Froggatt, K., \& Higginson, I. J. (2011). Interventions for improving palliative care for older people living in nursing care homes. The Cochrane Database of Systematic Reviews, 2011 CD007132. https://doi.org/10.1002/14651858.CD007132.pub2

Hargreaves, C., Tucker, S., Hughes, J., Hays, R., \& Challis, D. (2019). Specialist healthcare services for UK care home residents: A latent class analysis. Primary Health Care Research and Development, 20 , e132. https://doi.org/10.1017/S1463423619000586

Harris-Kojetin, L., Sengupta, M., Lendon, J. P., Rome, V., Valverde, R. \& Caffrey, C. (2019). Long-term care providers and services users in the United States, 2015-2016. National Center for Health Statistics. Vital Health Statistics, 3(43).

Harvey, P., Storer, M., Berlowitz, D. J., Jackson, B., Hutchinson, A., \& Lim, W. K. (2014). Feasibility and impact of a post-discharge geriatric evaluation and management service for patients from residential care: The Residential Care Intervention Program in the Elderly (RECIPE). BMC Geriatrics, 14, 48. https://doi.org/10.1186/1471-2318-14-48

Hex, N., Tuggey, J., Wright, D., \& Malin, R. (2015). Telemedicine in care homes in Airedale, Wharfedale and Craven. Clinical Governance, 20, 146-154. https://doi.org/10.1108/CGIJ-07-2015-0022
Hockley, J., \& Kinley, J. (2016). A practice development initiative supporting care home staff deliver high quality end-of-life care. International Journal of Palliative Nursing, 22, 474-481. https://doi. org/10.12968/ijpn.2016.22.10.474

Hockley, J., Watson, J., Oxenham, D., \& Murray, S. A. (2010). The integrated implementation of two end-of-life care tools in nursing care homes in the UK: An in-depth evaluation. Palliative Medicine, 24 828-838. https://doi.org/10.1177/0269216310373162

Hoffmann, T., Glasziou, P., Boutron, I., Milne, R., Perera, R., Moher, D., Altman, D., Barbour, V., Macdonald, H., Johnston, M., Lamb, S., Dixon-Woods, M., McCulloch, P., Wyatt, J., Chan, A., \& Michie, S. (2014). Better reporting of interventions: Template for intervention description and replication (TIDieR) checklist and guide. BMJ, 348, g1687. https://doi.org/10.1136/bmj.g1687

Hofmeyer, J., Leider, J. P., Satorius, J., Tanenbaum, E., Basel, D., \& Knudson, A. (2016). Implementation of telemedicine consultation to assess unplanned transfers in rural long-term care facilities, 2012-2015: A pilot study. Journal of the American Medical Directors Association, 17, 1006-1010. https://doi.org/10.1016/j.jamda.2016.06.014

Hui, E., \& Woo, J. (2002). Telehealth for older patients: The Hong Kong experience. Journal of Telemedicine and Telecare, 8, 39-41. https:// doi.org/10.1258/13576330260440808

Hui, E., Woo, J., Hjelm, M., Zhang, Y. T., \& Tsui, H. T. (2001). Telemedicine: A pilot study in nursing home residents. Gerontology, 47, 82-87. https://doi.org/10.1159/000052778

Hullick, C., Conway, J., Higgins, I., Hewitt, J., Dilworth, S., Holliday, E. \& Attia, J. (2016). Emergency department transfers and hospital admissions from residential aged care facilities: A controlled prepost design study. BMC Geriatrics, 16, 102. https://doi.org/10.1186/ s12877-016-0279-1

Hutchinson, A. F., Parikh, S., Tacey, M., Harvey, P. A., \& Lim, W. K. (2015). A longitudinal cohort study evaluating the impact of a geriatricianled residential care outreach service on acute healthcare utilisation. Age and Ageing, 44, 365-370. https://doi.org/10.1093/agein g/afu196

Hutt, E., Ruscin, J. M., Linnebur, S. A., Fish, D. N., Oman, K. S., Fink, R. M., Radcliff, T. A., Van Dorsten, B., Liebrecht, D., Fish, R., \& McNulty, M. C. (2011). A multifaceted intervention to implement guidelines did not affect hospitalization rates for nursing home-acquired pneumonia. Journal of the American Medical Directors Association, 12, 499-507. https://doi.org/10.1016/j.jamda.2010.03.011

Iliffe, S., Davies, S. L., Gordon, A. L., Schneider, J., Dening, T., Bowman, C., Gage, H., Martin, F. C., Gladman, J. R., Victor, C., Meyer, J., \& Goodman, C. (2016). Provision of NHS generalist and specialist services to care homes in England: Review of surveys. Primary Health Care Research and Development, 17(2), 122-137. https://doi. org/10.1017/S1463423615000250

Ingber, M. J., Feng, Z., Khatutsky, G., Wang, J. M., Bercaw, L. E., Zheng, N. T., Vadnais, A., Coomer, N. M., \& Segelman, M. (2017). Initiative to reduce avoidable hospitalizations among nursing facility residents shows promising results. Health Affairs, 36, 441-450. https://doi. org/10.1377/hlthaff.2016.1310

Jackson, N., \& Waters, E. (2005). Criteria for the systematic review of health promotion and public health interventions. Health Promotion International, 20, 367-374. https://doi.org/10.1093/heapro/dai022

Jafari, M., \& Ansari-Pour, N. (2019). Why, When and how to adjust your P values? Cell Journal, 20(4), 604-607. https://doi.org/10.22074/ cellj.2019.5992

Jensen, J. L., Marshall, E. G., Carter, A. J., Boudreau, M., Burge, F., \& Travers, A. H. (2016). Impact of a Novel Collaborative Long-Term Care -EMS Model: A before-and-after cohort analysis of an extended care paramedic program. Prehospital Emergency Care, 20, 111-116. https://doi.org/10.3109/10903127.2015.1051678

Joseph, A., \& Boult, C. (1998). Managed primary care of nursing home residents. Journal of the American Geriatrics Society, 46, 1152-1156. 
Kane, R. L., Flood, S., Bershadsky, B., \& Keckhafer, G. (2004). Effect of an innovative medicare managed care program on the quality of care for nursing home residents. Gerontologist, 44, 95-103. https://doi. org/10.1093/geront/44.1.95

Kane, R. L., Keckhafer, G., Flood, S., Bershadsky, B., \& Siadaty, M. S. (2003). The effect of Evercare on hospital use. Journal of the American Geriatrics Society, 51, 1427-1434. https://doi. org/10.1046/j.1532-5415.2003.51461.x

Kinley, J., Denton, L., \& Levy, J. (2018). Improving the approach to future care planning in care homes. International Journal of Palliative Nursing, 24, 576-583. https://doi.org/10.12968/ijpn.2018.24.12.576

Kinley, J., Stone, L., Dewey, M., Levy, J., Stewart, R., McCrone, P., Sykes, N., Hansford, P., Begum, A., \& Hockley, J. (2014). The effect of using high facilitation when implementing the Gold Standards Framework in Care Homes programme: A cluster randomised controlled trial. Palliative Medicine, 28, 1099-1109. https://doi.org/10.1177/02692 16314539785

Klaasen, K., Lamont, L., \& Krishnan, P. (2009). Setting a new standard of care in nursing homes. Canadian Nurse, 105, 24-30.

Kuhn, D. R., \& Forrest, J. M. (2012). Palliative care for advanced dementia: A pilot project in 2 nursing homes. American Journal of Alzheimer's Disease and Other Dementias, 27, 33-40. https://doi. org/10.1177/1533317511432732

Kunte, V., Johansen, M. L., \& Isenberg-Cohen, S. (2017). Improving longterm care residents' outcomes by educating nursing staff on endof-life communication. Journal of Hospice and Palliative Nursing, 19, 550-555. https://doi.org/10.1097/NJH.0000000000000386

Lacny, S., Zarrabi, M., Martin-Misener, R., Donald, F., Sketris, I., Murphy, A. L., DiCenso, A., \& Marshall, D. A. (2016). Cost-effectiveness of a nurse practitioner-family physician model of care in a nursing home: Controlled before and after study. Journal of Advanced Nursing, 72, 2138-2152. https://doi.org/10.1111/jan.12989

Lapane, K. L., Hughes, C. M., Christian, J. B., Daiello, L. A., Cameron, K. A., \& Feinberg, J. (2011). Evaluation of the fleetwood model of longterm care pharmacy. Journal of the American Medical Association, 12, 355-363. https://doi.org/10.1016/j.jamda.2010.03.003

Lapane, K. L., Hughes, C. M., Daiello, L. A., Cameron, K. A., \& Feinberg, J. (2011). Effect of a pharmacist-led multicomponent intervention focusing on the medication monitoring phase to prevent potential adverse drug events in nursing homes. Journal of the American Geriatrics Society, 59, 1238-1245. https://doi. org/10.1111/j.1532-5415.2011.03418.x

Lisk, R., Yeong, K., Nasim, A., Baxter, M., Mandal, B., Nari, R., \& Dhakam, Z. (2012). Geriatrician input into nursing homes reduces emergency hospital admissions. Archives of Gerontology and Geriatrics, 55, 331337. https://doi.org/10.1016/j.archger.2011.10.014

Luk, J. K. H., \& Chan, F. H. W. (2017). End-of-life care for advanced dementia patients in residential care home-a Hong Kong perspective. Annals of Palliative Medicine, 7, 359-364. https://doi.org/10.21037/ apm.2017.08.13

Luk, J. K. H., Pau, M. M. L., Chan, F. H. W., \& Yu, C. (2002). Outreach geriatric services to private old age homes in Hong Kong West cluster. Journal of the Hong Kong Geriatrics Society, 11, 5-10.

Mason, S., O'Keeffe, C., Knowles, E., Bradburn, M., Campbell, M., Coleman, P., Stride, C., O'Hara, R., Rick, J., \& Patterson, M. (2012). A pragmatic quasi-experimental multi-site community intervention trial evaluating the impact of Emergency Care Practitioners in different UK health settings on patient pathways (NEECaP Trial). Emergency Medicine Journal, 29, 47-53. https://doi.org/10.1136/ emj.2010.103572

McAiney, C. A., Haughton, D., Jennings, J., Farr, D., Hillier, L., \& Morden, P. (2008). A unique practice model for Nurse Practitioners in longterm care homes. Journal of Advanced Nursing, 62, 562-571. https:// doi.org/10.1111/j.1365-2648.2008.04628.x.

McAndrew, R. M., Grabowski, D. C., Dangi, A., \& Young, G. J. (2016). Prevalence and patterns of potentially avoidable hospitalizations in the US long-term care setting. International Journal for Quality in Health Care, 23, 104-109. https://doi.org/10.1093/intqhc/mzv110

McKee, H., Miller, R., Cuthbertson, S. C., \& Scott, M. G. (2016). Nursing Home Outreach Clinics show an improvement in patient safety and reduction in hospital admissions in residents with chronic conditions. European Journal for Person Centered Healthcare, 4, 650-655. https://doi.org/10.5750/ejpch.v4i4.1178

McKenzie, J. E., \& Brennan, S. E. (2019). Chapter 12: Synthesizing and presenting findings using other methods. In J. P. T. Higgins, J. Thomas, J. Chandler, M. Cumpston, T. Li, M. J. Page, \& V. A. Welch (Eds.), Cochrane handbook for systematic reviews of interventions, 2nd ed. John Wiley \& Sons.

Montalto, M. (2001). Hospital in the nursing home. Treating acute hospital problems in nursing home residents using a Hospital in the Home model. Australian Family Physician, 30, 1010-1012.

Moore, K. J., Candy, B., Davis, S., Gola, A., Harrington, J., Kupeli, N., Vickerstaff, V., King, M., Leavey, G., Nazareth, I., Omar, R. Z., Jones, L., \& Sampson, E. L. (2017). Implementing the compassion intervention, a model for integrated care for people with advanced dementia towards the end of life in nursing homes: a naturalistic feasibility study. British Medical Journal Open, 7, e015515. https://doi. org/10.1136/bmjopen-2016-015515

Mulrow, C. D., Gerety, M. B., Kanten, D., Cornell, J. E., DeNino, L. A., Chiodo, L., Aguilar, C., O'Neil, M. B., Rosenberg, J., \& Solis, R. M. (1994). A randomized trial of physical rehabilitation for very frail nursing home residents. Journal of the American Medical Association, 271, 519-524. https://doi.org/10.1001/jama.1994.0351031004 9037.

National Health Service. (2019). The NHS Long Term Plan. Retrieved October 24, 2020, from https://www.longtermplan.nhs.uk/wpcontent/uploads/2019/01/nhs-long-term-plan-june-2019.pdf

New South Wales Aged Care Roundtable. (2019). Joint report on avoidable hospitalisations from residential aged care facilities in NSW and delayed discharge. NSW Aged Care Roundtable. Retrieved August 7, 2021, from https://www.nswnma.asn.au/wp-content/ uploads/2019/09/NSW-Aged-Care-Roundtable-2019-LR-FINAL. pdf

NHS England. (2015). Quick guide: Clinical input into care homes. Retrieved August 7, 2021, from https://www.nhs.uk/NHSEngland/ keogh-review/Documents/quick-guides/Quick-Guide-clinicalinput-to-care-homes.pdf

NHS England. (2020). The framework for enhanced health in care homes. Retrieved October 24, 2020, from https://www.england.nhs.uk/ wp-content/uploads/2020/03/the-framework-for-enhanced-healt h-in-care-homes-v2-0.pdf

Nuffield Trust. (2020). Care home bed availability. Retrieved August 7, 2021, from https://www.nuffieldtrust.org.uk/resource/carehome-bed-availability

O'Cathain, A., Croot, L., Duncan, E., Rousseau, N., Sworn, K., Turner, K. M., Yardley, L., \& Hoddinott, P. (2019). Guidance on how to develop complex interventions to improve health and healthcare. British Medical Journal, 9, e029954. https://doi.org/10.1136/bmjop en-2019-029954

Olsson, I. N., Curman, B., \& Engfeldt, P. (2010). Patient focused drug surveillance of elderly patients in nursing homes. Pharmacoepidemiology and Drug Safety, 19, 150-157. https://doi.org/10.1002/pds.1891

Ono, M., Miyauchi, S., Edzuki, Y., Saiki, K., Fukuda, H., Tonai, M., Magilvy, J. K., \& Murashima, S. (2015). Japanese nurse practitioner practice and outcomes in a nursing home. International Nursing Review, 62, 275-279. https://doi.org/10.1111/inr.12158

O'Sullivan, R., Murphy, A., O'Caoimh, R., Cornally, N., Svendrovski, A., Daly, B., Fizgerald, C., Twomey, C., McGlade, C., \& Molloy, D. W. (2016). Economic (gross cost) analysis of systematically implementing a programme of advance care planning in three Irish nursing homes. BMC Research Notes, 9, 237. https://doi.org/10.1186/s1310 4-016-2048-9 
Ouslander, J. G., Lamb, G., Tappen, R., Herndon, L., Diaz, S., Roos, B. A., Grabowski, D. C., \& Bonner, A. (2011). Interventions to reduce hospitalizations from nursing homes: Evaluation of the INTERACT II collaborative quality improvement project. Journal of the American Geriatrics Society, 59, 745-753. https://doi. org/10.1111/j.1532-5415.2011.03333.x

Page, M. J., McKenzie, J. E., Bossuyt, P. M., Boutron, I., Hoffmann, T. C., Mulrow, C. D., Shamseer, L., Tetzlaff, J. M., Akl, E. A., Brennan, S. E., Chou, R., Glanville, J., Grimshaw, J. M., Hróbjartsson, A., Lalu, M. M., Li, T., Loder, E. W., Mayo-Wilson, E., McDonald, S., ... Moher, D. (2021). The PRISMA 2020 statement: an updated guideline for reporting systematic reviews. British Medical Journal, 372, n71. https://doi.org/10.1136/bmj.n71

Pain, T., Stainkey, L., \& Chapman, S. (2014). AgedCare+GP: Description and evaluation of an in-house model of general practice in a residential aged-care facility. Australian Journal of Primary Health, 20, 224-227. https://doi.org/10.1071/PY12151

Pedersen, L. H., Gregersen, M., Barat, I., \& Damsgaard, E. M. (2016). Early geriatric follow-up after discharge reduces readmissions? A quasirandomised controlled trial. European Geriatric Medicine, 7, 443448. https://doi.org/10.1016/j.eurger.2016.03.009

Pedersen, L. H., Gregersen, M., Barat, I., \& Damsgaard, E. M. (2018). Early geriatric follow-up visits to nursing home residents reduce the number of readmissions: A quasi-randomised controlled trial. European Geriatric Medicine, 9, 329-337. https://doi.org/10.1007/ s41999-018-0045-3

Pitkälä, K. H., Juola, A. L., Kautiainen, H., Soini, H., Finne-Soveri, U. H., Bell, J. S., \& Björkman, M. (2014). Education to reduce potentially harmful medication use among residents of assisted living facilities: A randomized controlled trial. Journal of the American Medical Directors Association, 15, 892-898. https://doi.org/10.1016/j. jamda.2014.04.002

Potter, K., Flicker, L., Page, A., \& Etherton-Beer, C. (2016). Deprescribing in frail older people: A randomised controlled trial. PLoS One, 11 e0149984. https://doi.org/10.1371/journal.pone.0149984

Public Health England (2017). The role of care homes in end of life care Briefing 2-Place and cause of death for permanent and temporary residents of care homes. Retrieved August 7, 2021, from https:// assets.publishing.service.gov.uk/government/uploads/system/ uploads/attachment_data/file/828122/Briefing_2_Place_and_ cause_of_death_for_permanent_and_temporary_residents_of_ care_homes.pdf

Rantz, M. J., Birtley, N. M., Flesner, M., Crecelius, C., \& Murray, C. (2017). Call to action: APRNs in U.S. nursing homes to improve care and reduce costs. Nursing Outlook, 65, 689-696. https://doi. org/10.1016/j.outlook.2017.08.011

Rantz, M. J., Popejoy, L., Vogelsmeier, A., Galambos, C., Alexander, G., Flesner, M., Crecelius, C., Ge, B., \& Petroski, G. (2017) Successfully reducing hospitalizations of nursing home residents: Results of the Missouri quality initiative. Journal of the American Medical Association, 18, 960-966. https://doi.org/10.1016/j. jamda.2017.05.027

(2020). Review Manager (RevMan) [Computer program]. Version 5.4. The Cochrane Collaboration.

Reymond, L., Israel, F. J., \& Charles, M. A. (2011). A residential aged care end-of-life care pathway (RAC EoLCP) for Australian aged care facilities. Australian Health Review, 35, 350-356. https://doi. org/10.1071/AH10899

Richardson, S. J., Carroll, C. B., Close, J., Gordon, A. L., O'Brien, J., Quinn, T. J., Rochester, L., Sayer, A. A., Shenkin, S. D., van der Velde, N., Woo, J., \& Witham, M. D. (2020). Research with older people in a world with COVID-19: Identification of current and future priorities, challenges and opportunities. Age and Ageing, 49, 901-906. https://doi.org/10.1093/ageing/afaa149

Roberts, M. S., Stokes, J. A., King, M. A., Lynne, T. A., Purdie, D. M., Glasziou, P. P., Wilson, D. A., McCarthy, S. T., Brooks, G. E., de
Looze, F. J., \& Del Mar, C. B. (2001). Outcomes of a randomized controlled trial of a clinical pharmacy intervention in 52 nursing homes. British Journal of Clinical Pharmacology, 51, 257-265. https:// doi.org/10.1046/j.1365-2125.2001.00347.x

Rolland, Y., Mathieu, C., Piau, C., Cayla, F., Bouget, C., Vellas, B., \& de Souto Barreto, P. (2016). Improving the quality of care of long-stay nursing home residents in France. Journal of the American Geriatrics Society, 64, 193-199. https://doi.org/10.1111/jgs.13874

Romøren, M., Gjelstad, S., \& Lindbæk, M. (2017). A structured training program for health workers in intravenous treatment with fluids and antibiotics in nursing homes: A modified stepped-wedge cluster-randomised trial to reduce hospital admissions. PLoS One, 12, e0182619. https://doi.org/10.1371/journal.pone.0182619

Russell, S. (2017). Living well in an aged care home. Research Matters: Melbourne. Retrieved August 7, 2021, from http://www.agedc arematters.net.au/wp-content/uploads/2017/10/LivingWell AgedCareHome-1.pdf

Rutter, D., Francis, J., Coren, E., \& Fisher, M. (2010). SCIE systematic research reviews: Guidelines, 2nd ed. Social Care Institute for Excellence.

Sackley, C. M., Walker, M. F., Burton, C. R., Watkins, C. L., Mant, J., Roalfe, A. K., Wheatley, K., Sheehan, B., Sharp, L., Stant, K. E., Fletcher-Smith, J., Steel, K., Barton, G. R., Irvine, L., \& Peryer, G. (2016). An Occupational Therapy intervention for residents with stroke-related disabilities in UK Care Homes (OTCH): Cluster randomised controlled trial with economic evaluation. Health Technology Assessment, 20(15), 1-138. https://doi.org/10.3310/ hta20150

Salles, N., Lafargue, A., Cressot, V., Glenisson, L., Barateau, M., Thiel, E., Doutre, M. S., Bourdin, N., Prigent, A. M., Libert, K., Dupuy, M., Caubet, C., \& Raynal, F. (2017). Global geriatric evaluation is feasible during interactive telemedicine in nursing homes. European Research in Telemedicine, 6, 59-65. https://doi.org/10.1016/j. eurtel.2017.06.002

Sankaran, S., Kenealy, T., Adair, A., Adair, V., Coster, H., Whitehead, N., Sheridan, N., Parsons, M., Marshall, E., Bailey, L., Price, C., Crombie, D., \& Rea, H. (2010). A complex intervention to support 'rest home' care: A pilot study. New Zealand Medical Journal, 123, 41-53.

Santosaputri, E., Laver, K., \& To, T. (2018). Efficacy of interventions led by staff with geriatrics expertise in reducing hospitalisation in nursing home residents: A systematic review. Australasian Journal on Ageing, 38, 5-14. https://doi.org/10.1111/ajag.12593

Schubert, C. C., Myers, L. J., Allen, K., \& Counsell, S. R. (2016). Implementing Geriatric Resources for Assessment and Care of Elders Team Care in a Veterans Affairs Medical Center: Lessons learned and effects observed. Journal of the American Geriatrics Society, 64, 1503-1509. https://doi.org/10.1111/jgs.14179

Schünemann, H. J., Higgins, J. P. T., Vist, G. E., Glasziou, P., Akl, E. A., Skoetz, N., \& Guyatt, G. H. (2021). Chapter 14: Completing 'Summary of findings' tables and grading the certainty of the evidence. In J. P. T. Higgins, J. Thomas, J. Chandler, M. Cumpston, T. Li, M. J. Page, \& V. A. Welch (Eds.), Cochrane handbook for systematic reviews of interventions version 6.2 (updated February 2021). Cochrane, 2021. Retrieved July 21, 2021, from www.training.cochr ane.org/handbook

Shah, M. N., Wasserman, E. B., Gillespie, S. M., Wood, N. E., Wang, H., Noyes, K., Nelson, D., Dozier, A., \& McConnochie, K. M. (2015). High-intensity telemedicine decreases emergency department use for ambulatory care sensitive conditions by older adult senior living community residents. Journal of the American Medical Association, 16, 1077-1081. https://doi.org/10.1016/j. jamda.2015.07.009

Smith, D., \& Brown, S. (2017). Integrating a palliative care approach into nursing care homes for older people. International Journal of Palliative Nursing, 23, 511-515. https://doi.org/10.12968/ ijpn.2017.23.10.511 
Social Care Institute for Excellence. (2017). End of life care in a care home. Retrieved August 7, 2021, from https://www.scie.org.uk/ person-centred-care/older-people-care-homes/end-of-life-care

Stern, A., Mitsakakis, N., Paulden, M., Alibhai, S., Wong, J., Tomlinson, G., Brooker, A. S., Krahn, M., \& Zwarenstein, M. (2014). Pressure ulcer multidisciplinary teams via telemedicine: A pragmatic cluster randomized stepped wedge trial in long term care. BMC Health Services Research, 14, 83. https://doi.org/10.1186/1472-6963-14-83

Street, M., Considine, J., Livingston, P., Ottmann, G., \& Kent, B. (2015). In-reach nursing services improve older patient outcomes and access to emergency care. Australasian Journal on Ageing, 34, 115120. https://doi.org/10.1111/ajag.12137

Tena-Nelson, R., Santos, K., Weingast, E., Amrhein, S., Ouslander, J., \& Boockvar, K. (2012). Reducing potentially preventable hospital transfers: Results from a thirty nursing home collaborative. Journal of the American Medical Directors Association, 13, 651-656. https:// doi.org/10.1016/j.jamda.2012.06.011

Teo, W. S., Raj, A. G., Tan, W. S., Ng, C. W., Heng, B. H., \& Leong, I. Y. (2014). Economic impact analysis of an end-of-life programme for nursing home residents. Palliative Medicine, 28, 430-437. https:// doi.org/10.1177/0269216314526270

Thiruchelvam, K., Hasan, S. S., Wong, P. S., \& Kairuz, T. (2017). Residential aged care medication review to improve the quality of medication use: A systematic review. Journal of the American Medical Directors Association, 18, 87.e1-87.e14. https://doi.org/10.1016/j. jamda.2016.10.004

Thomas, B. H., Ciliska, D., Dobbins, M., \& Micucci, S. (2004). A process for systematically reviewing the literature: Providing the research evidence for public health nursing interventions. Worldviews on Evidence-Based Nursing, 1, 176-184. https://doi. org/10.1111/j.1524-475X.2004.04006.x

Tucker, S., Buck, D., Roe, B., Hughes, J., \& Challis, D. (2019). Hospital admissions and place of death of residents of care homes receiving specialist healthcare services: Protocol for a systematic review. Journal of Advanced Nursing, 75, 443-451. https://doi.org/10.1111/ jan.13866

Unroe, K. T., Fowler, N. R., Carnahan, J. L., Holtz, L. R., Hickman, S. E., Effler, S., Evans, R., Frank, K. I., Ott, M. L., \& Sachs, G. (2018). Improving nursing facility care through an innovative payment demonstration project: Optimizing patient transfers, impacting medical quality, and improving symptoms: Transforming institutional care phase 2. Journal of the American Geriatrics Society, 66 , 1625-1631. https://doi.org/10.1111/jgs.15462

Wade, V., Whittaker, F., \& Hamlyn, J. (2015). An evaluation of the benefits and challenges of video consulting between general practitioners and residential aged care facilities. Journal of Telemedicine and Telecare, 21, 490-493. https://doi.org/10.1177/1357633X15 611771
Walker, G. M., Armstrong, S., Gordon, A. L., Gladman, J., Robertson, K., Ward, M., Conroy, S., Arnold, G., Darby, J., Frowd, N., Williams, W. Knowles, S., \& Logan, P. A. (2016). The Falls In Care Home study: A feasibility randomized controlled trial of the use of a risk assessment and decision support tool to prevent falls in care homes. Clinical Rehabilitation, 30, 972-983. https://doi.org/10.1177/02692 15515604672

Wallerstedt, S. M., Kindblom, J. M., Nylén, K., Samuelsson, O., \& Strandell, A. (2014). Medication reviews for nursing home residents to reduce mortality and hospitalization: Systematic review and meta-analysis. British Journal of Clinical Pharmacology, 78, 488-497. https://doi. org/10.1111/bcp.12351

Ward, J. A., Harden, M., Gibson, R. E., \& Byles, J. E. (2010). A cluster randomised controlled trial to prevent injury due to falls in a residential aged care population. Medical Journal of Australia, 192, 319-322. https://doi.org/10.5694/j.1326-5377.2010.tb03530.x

Wiggins, N., Droney, J., Mohammed, K., Riley, J., \& Sleeman, K. E. (2019). Understanding the factors associated with patients with dementia achieving their preferred place of death: A retrospective cohort study. Age and Ageing, 48, 433-439. https://doi.org/10.1093/agein g/afz015

Winstanley, L., \& Brennan, W. (2007). Advanced practice and support in prescribing and medicine management for care homes. Journa of Care Services Management, 1, 233-244. https://doi.org/10.1179/ csm.2007.1.3.233

Wolters, A., Santos, F., Lloyd, T., Lilburne, C., \& Steventon, A. (2019). Emergency admissions to hospital from care homes: how often and what for? The Health Foundation.

Zermansky, A. G., Alldred, D. P., Petty, D. R., Raynor, D. K., Freemantle, N., Eastaugh, J., \& Bowie, P. (2006). Clinical medication review by a pharmacist of elderly people living in care homes-randomised controlled trial. Age and Ageing, 35, 586-591. https://doi.org/10.1093/ ageing/afl075

\section{SUPPORTING INFORMATION}

Additional supporting information may be found in the online version of the article at the publisher's website.

How to cite this article: Buck, D., Tucker, S., Roe, B., Hughes, J., \& Challis, D. (2021). Hospital admissions and place of death of residents of care homes receiving specialist healthcare services: A systematic review without metaanalysis. Journal of Advanced Nursing, 00, 1-32. https://doi. org/10.1111/jan.15043 
The Journal of Advanced Nursing (JAN) is an international, peer-reviewed, scientific journal. JAN contributes to the advancement of evidence-based nursing, midwifery and health care by disseminating high quality research and scholarship of contemporary relevance and with potential to advance knowledge for practice, education, management or policy. JAN publishes research reviews, original research reports and methodological and theoretical papers.

For further information, please visit JAN on the Wiley Online Library website: www.wileyonlinelibrary.com/journal/jan

Reasons to publish your work in JAN:

- High-impact forum: the world's most cited nursing journal, with an Impact Factor of 2.561 - ranked 6/123 in the 2019 ISI Journal Citation Reports $\odot$ (Nursing; Social Science).

- Most read nursing journal in the world: over 3 million articles downloaded online per year and accessible in over 10,000 libraries worldwide (including over 6,000 in developing countries with free or low cost access).

- Fast and easy online submission: online submission at http://mc.manuscriptcentral.com/jan.

- Positive publishing experience: rapid double-blind peer review with constructive feedback.

- Rapid online publication in five weeks: average time from final manuscript arriving in production to online publication.

- Online Open: the option to pay to make your article freely and openly accessible to non-subscribers upon publication on Wiley Online Library, as well as the option to deposit the article in your own or your funding agency's preferred archive (e.g. PubMed). 